\title{
Estimation of stellar atmospheric parameters from SDSS/SEGUE spectra
}

\author{
P. Re Fiorentin ${ }^{1}$, C. A. L. Bailer-Jones ${ }^{1}$, Y. S. Lee ${ }^{2}$, T. C. Beers ${ }^{2}$, T. Sivarani ${ }^{2}$, R. Wilhelm ${ }^{3}$, \\ C. Allende Prieto ${ }^{4}$, and J. E. Norris ${ }^{5}$ \\ ${ }^{1}$ Max Planck Institut für Astronomie, Königstuhl 17, 69117 Heidelberg, Germany \\ e-mail: fiorent@mpia.de \\ 2 Department of Physics \& Astronomy, CSCE: Center for the Study of Cosmic Evolution, and JINA: Joint Institute for Nuclear \\ Astrophysics, Michigan State University, East Lansing, MI 48824, USA \\ 3 Department of Physics, Texas Tech University, Lubbock, TX 79409, USA \\ 4 Department of Astronomy, University of Texas, Austin, TX 78712, USA \\ 5 Research School of Astronomy and Astrophysics, Australian National University, Weston, ACT 2611, Australia
}

Received 20 February 2007 / Accepted 8 March 2007

\section{ABSTRACT}

\begin{abstract}
We present techniques for the estimation of stellar atmospheric parameters $\left(T_{\text {eff }}, \log g,[\mathrm{Fe} / \mathrm{H}]\right)$ for stars from the SDSS/SEGUE survey. The atmospheric parameters are derived from the observed medium-resolution $(R=2000)$ stellar spectra using non-linear regression models trained either on (1) pre-classified observed data or (2) synthetic stellar spectra. In the first case we use our models to automate and generalize parametrization produced by a preliminary version of the SDSS/SEGUE Spectroscopic Parameter Pipeline (SSPP). In the second case we directly model the mapping between synthetic spectra (derived from Kurucz model atmospheres) and the atmospheric parameters, independently of any intermediate estimates. After training, we apply our models to various samples of SDSS spectra to derive atmospheric parameters, and compare our results with those obtained previously by the SSPP for the same samples. We obtain consistency between the two approaches, with RMS deviations on the order of $150 \mathrm{~K}$ in $T_{\text {eff }}, 0.35 \mathrm{dex}$ in $\log g$, and $0.22 \mathrm{dex}$ in $[\mathrm{Fe} / \mathrm{H}]$.

The models are applied to pre-processed spectra, either via Principal Component Analysis (PCA) or a Wavelength Range Selection (WRS) method, which employs a subset of the full 3850-9000 ̊ spectral range. This is both for computational reasons (robustness and speed), and because it delivers higher accuracy (better generalization of what the models have learned). Broadly speaking, the PCA is demonstrated to deliver more accurate atmospheric parameters when the training data are the actual SDSS spectra with previously estimated parameters, whereas WRS appears superior for the estimation of $\log g$ via synthetic templates, especially for lower signal-to-noise spectra. From a subsample of some 19000 stars with previous determinations of the atmospheric parameters, the accuracies of our predictions (mean absolute errors) for each parameter are $T_{\text {eff }}$ to $170 / 170 \mathrm{~K}, \log g$ to $0.36 / 0.45 \mathrm{dex}$, and $[\mathrm{Fe} / \mathrm{H}]$ to $0.19 / 0.26 \mathrm{dex}$, for methods (1) and (2), respectively. We measure the intrinsic errors of our models by training on synthetic spectra and evaluating their performance on an independent set of synthetic spectra. This yields RMS accuracies of $50 \mathrm{~K}$, 0.02 dex, and 0.03 dex on $T_{\text {eff }}, \log g$, and [Fe/H], respectively.

Our approach can be readily deployed in an automated analysis pipeline, and can easily be retrained as improved stellar models and synthetic spectra become available. We nonetheless emphasise that this approach relies on an accurate calibration and pre-processing of the data (to minimize mismatch between the real and synthetic data), as well as sensible choices concerning feature selection. From an analysis of cluster candidates with available SDSS spectroscopy (M 15, M 13, M 2, and NGC 2420), and assuming the age, metallicity, and distances given in the literature are correct, we find evidence for small systematic offsets in $T_{\text {eff }}$ and/or log $g$ for the parameter estimates from the model trained on real data with the SSPP. Thus, this model turns out to derive more precise, but less accurate, atmospheric parameters than the model trained on synthetic data.
\end{abstract}

Key words. surveys - methods: data analysis - methods: statistical - stars: fundamental parameters

\section{Introduction}

The nature of the stellar populations of the Milky Way galaxy remains an important issue for astrophysics, because it addresses the question of galaxy formation and evolution and the evolution of the chemical elements. To date, however, studies of the stellar populations, kinematics, and chemical abundances in the Galaxy have mostly been limited by small number statistics.

Fortunately, this state of affairs is rapidly changing. The Sloan Digital Sky Survey (SDSS; York et al. 2000) has imaged over 8000 square degrees of the northern Galactic cap (above $|b|=40^{\circ}$ ) in the ugriz photometric system for some 100 million stars. Imaging data are produced simultaneously
(Fukugita et al. 1996; Gunn et al. 1998, 2006; Hogg et al. 2001; Abazajian et al. 2005; Adelman-McCarthy et al. 2007) and processed through pipelines to measure photometric and astrometric properties (Lupton et al. 1987; Stoughton et al. 2002; Smith et al. 2002; Tucker et al. 2002; Pier et al. 2003; Ivézic et al. 2004) and to select targets for spectroscopic follow-up. Of even greater importance, some 200000 medium-resolution stellar spectra have been obtained during the course of SDSS-I (the original survey).

The SDSS-II project, which includes SEGUE (Sloan Extension for Galactic Understanding and Exploration), is obtaining some 3500 square degrees of additional imaging data at lower Galactic latitudes, in order to better explore the interface between the thick-disk and halo populations between 
0.5-4 kpc from the Galactic plane. SEGUE will obtain some 250000 medium-resolution spectra of stars in the Galaxy in the magnitude range $14.0 \leq g \leq 20.5$ in 200 fields covering the sky visible from the northern hemisphere (Apache Point Observatory, New Mexico). The targets are selected based on the photometry, and are chosen to provide tracers of the structure, chemical evolution, and stellar content of the Milky Way from 0.5 to $100 \mathrm{kpc}$ from the Sun. Taken together, the stellar database from SDSS-I and SEGUE provides an unprecedented opportunity for developing better understanding of the properties of the Milky Way.

Of special importance to achieve these goals is the determination of intrinsic stellar physical properties, such as masses, ages, and elemental abundances. The first step toward achieving this goal is to estimate the atmospheric parameters for these stars. A number of early studies (e.g., Gulati et al. 1996; Bailer-Jones et al. 1997, 1998; Bailer-Jones 2000; Snider et al. 2001; Willemsen et al. 2005) have demonstrated that non-linear regression models can be robust and precise classifiers of stellar spectra, either when trained on pre-classified observed data or on synthetic stellar spectra. In this paper we further explore the capability of these techniques to estimate $T_{\text {eff }}, \log g$, and $[\mathrm{Fe} / \mathrm{H}]$ specifically for SDSS/SEGUE spectroscopy and photometry. Alternative procedures are described by Allende Prieto et al. (2006), Lee et al. (2006), and Lee et al. (2007).

In this paper we explore three approaches in which either synthetic ("S") or real ("R") data are used for training and/or testing. With SS (training and testing on synthetic data), estimates of the atmospheric parameters are obtained from the model spectra, and the application is merely a test of the limits of the pre-processing/regression model. In RR (training and testing on real data), we use a set of pre-parametrized SEGUE spectra, in this case from a preliminary version of the SDSS/SEGUE Spectroscopic Parameter Pipeline (SSPP). Our model automates and, more importantly, generalizes these parametrizations. The model performance is evaluated on a separate set of data obtained from SDSS/SEGUE. SR is a model trained on synthetic data and applied to real data, thus allowing us to directly determine the atmospheric parameters without using an intermediate parametrization model. As we have no definitive "true" values against which to compare our parametrizations, we instead compare the results of the SR and RR models to parameters estimated by the SSPP (on a set of data not used to train RR). Of course, in both the SR and RR cases the derived parameters are based on a set of model atmospheres - the difference is how the atmospheric parameters are derived from them.

The layout of this paper is as follows. In Sect. 2 we describe the spectroscopic and photometric data from which preliminary estimates of the atmospheric parameters were obtained. Our regression model is described in Sect. 3. In Sect. 4 we discuss the advantages of dimensionality reduction via Principal Component Analysis, as well as from wavelength ("feature") selection. The results of the application of our methods using the SS, RR, and SR approaches are discussed in Sect. 5. An independent assessment of the accuracy (and calibration) of our models is provided in Sect. 6, where we estimate the atmospheric parameters of stars in several Galactic globular and open clusters. Finally, in Sect. 7 we provide our conclusions.

\section{Data}

In this section we discuss the SDSS/SEGUE spectra and the synthetic spectra that were constructed in order to build our models.

\subsection{Sample of real spectra}

Stellar spectra from SDSS/SEGUE cover the wavelength range 3850-9000 $\AA$ at a resolving power $R=\lambda / \Delta \lambda \simeq 2000$. The spectra are wavelength calibrated and approximately flux corrected using procedures described in Stoughton et al. (2002). For the purpose of our work, we first rebin to a final dispersion of $1.0 \AA /$ pixel in the blue region $3850-6000 \AA$, and $1.5 \AA /$ pixel in the red region $6000-9000 \AA$. Since the spectrophotometric corrections applied to these spectra are only approximate, we remove the continuum via an automated, iterative procedure (described in Sect. 2.2).

We have selected a sample of 38731 stellar spectra for stars in regions of low reddening, and for which atmospheric parameter estimates of effective temperature, gravity, and metallicity ( $\left.T_{\text {eff }}, \log g,[\mathrm{Fe} / \mathrm{H}]\right)$ have been obtained previously using the combination of procedures described in the SSPP (Lee et al. 2007), including several that rely on the available ugriz photometry. These methods include chi-square minimization with respect to synthetic spectral templates, neural networks, autocorrelation analysis, and a variety of line index calculations based on previous calibrations with respect to known standard stars. Estimates of the likely external errors in spectroscopic parameter determinations are in the process of being obtained by comparison with a number of previously available stellar spectroscopic libraries, as well as with high-resolution spectroscopy of over 100 SDSS/SEGUE stars. The use of multiple methods allows for empirical determinations of the internal errors for each parameter. However, we remark that at present the parameters from SSPP are inhomogeneously assembled, in the sense that we are still in the process of exploring which techniques are optimal over the parameter ranges which we study. This situation will change in the near future, when the techniques involved in the SSPP can be evaluated more fully, and are used to produce a meaningful weighted average.

Radial velocities estimated by the SSPP are used to reduce all spectra to a common radial velocity zero point.

\subsection{Sample of synthetic spectra}

In recent years a number of new atmospheric models covering a wide range of atmospheric parameters have become available. Here we make use of a set of 1816 synthetic spectra calculated from Kurucz's NEWODF models (Castelli \& Kurucz 2003) with solar abundances by Asplund et al. (2005), including $\mathrm{H}_{2} \mathrm{O}$ opacities, an improved set of $\mathrm{TiO}$ lines, and no convective overshoot (Castelli et al. 1997). All pertinent molecular species are included in these models, even those whose features have minor strength in the wavelength range covered by the SDSS spectra. The synthetic spectra are generated using the turbospectrum synthesis code (Alvarez \& Plez 1998), and employ line broadening according to the prescription of Barklem \& O'Mara (1998). The linelists used come from a variety of sources. Updated atomic lines are taken mainly from the VALD database (Kupka et al. 1999). The molecular species CH, CN, and $\mathrm{OH}$ are provided by B. Plez (see Plez \& Cohen 2005), while the $\mathrm{NH}, \mathrm{C}_{2}$ molecules are from the Kurucz linelists (see http: //kurucz . harvard . edu/LINELISTS/LINESMOL/).

Note that, at present, the linelists used to generate the synthetic spectra do not include all of the interesting molecular species, in particular, the $\mathrm{MgH}$ and $\mathrm{CaH}$ features. We plan to include these molecules in an updated version of our synthetic spectra, which is now under construction. 

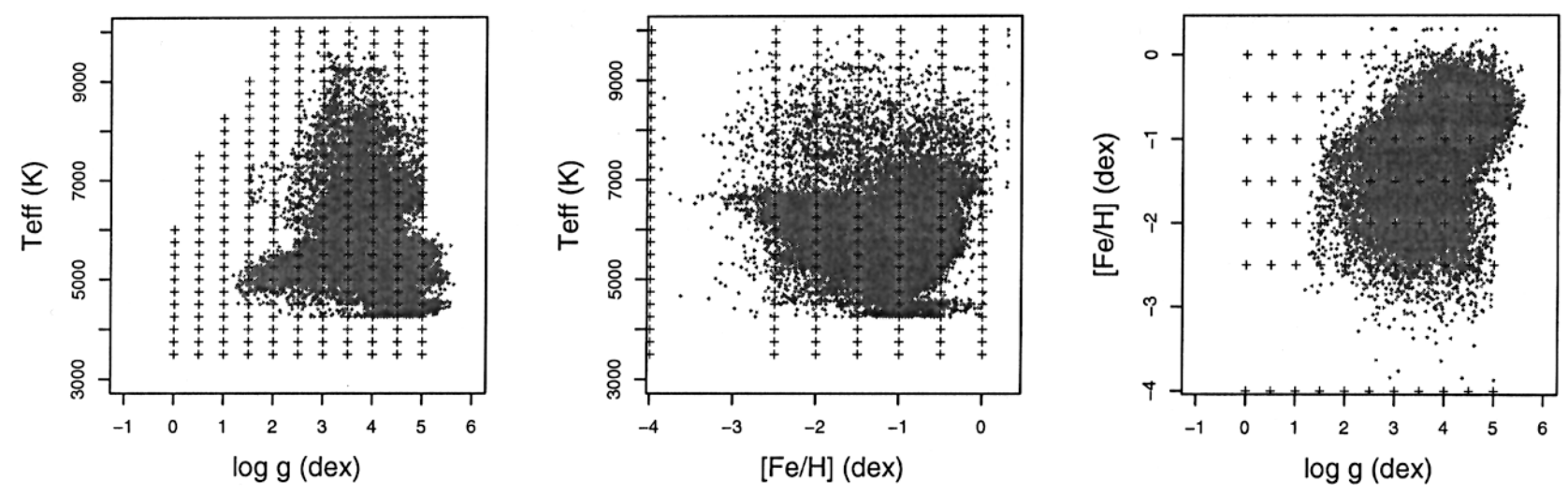

Fig. 1. The grid of stellar atmospheric parameters $T_{\text {eff }}, \log g$, and $[\mathrm{Fe} / \mathrm{H}]$. The synthetic parameters (plus symbols) are presented in comparison with previously estimated atmospheric parameters (dots) for 38731 SDSS/SEGUE spectra.

Our grids span the parameter ranges [3500, 10000$] \mathrm{K}$ in $T_{\text {eff }}$ (27 values, stepsize of $250 \mathrm{~K}),[0,5]$ dex in $\log g$ (11 values in 0.5 dex steps), and $[-4.0,0.0]$ dex in $[\mathrm{Fe} / \mathrm{H}](7$ values, stepsize between 0.5 dex and 1.5 dex; there is gap in the grid between $[\mathrm{Fe} / \mathrm{H}]=-2.5$ and -4.0$)$. The synthetic spectra are similarly divided into blue and red regions, and the same dispersion correction and flux "calibration" (i.e. instrument modeling) were applied to match the real SDSS/SEGUE spectra. Figure 1 shows the grid of the available parameters. The data used cover the full input range provided, $3850-9000 \AA$, in 4152 individual data bins. It should also be noted that we have not implemented any procedure to account for the inevitable presence of telluric lines, in particular near the location of the calcium triplet. At present, new reductions procedures for SDSS spectra are being explored to minimize the impact of telluric lines in this region.

The continuum is removed by dividing the spectrum by an iterative fifth-order polynomial fit of the spectrum. This is done separately for the blue and red regions. In the following we exclude the red region $6000-6500 \AA$, because we found that the synthetic spectra do not properly model the real ones. This discrepancy may be due in part to instrumental signatures in this spectral region, which corresponds to the wavelengths where the dichroic used in the dual-arm SDSS spectrographs split the incoming photons into the blue and red arms.

\section{Non-linear regression model}

We implement a flexible method of regression that provides a global non-linear mapping between a set of inputs (the stellar spectrum $\boldsymbol{x}_{i}$ ) and a set of outputs (the stellar atmospheric parameters, $\left.\boldsymbol{s}=\left\{T_{\text {eff }}, \log g,[\mathrm{Fe} / \mathrm{H}]\right\}\right)$

$\boldsymbol{s}(p)=f\left(\sum_{i} w_{i} \boldsymbol{x}_{i p}\right)$

where $p$ denotes the $p$ th flux vector (star) and $w_{i}$ the set of weights that characterise the regression model (Bailer-Jones 2000). To reduce the dynamical range of $T_{\text {eff }}$ and to better represent the uncertainties we use $\log T_{\text {eff }}$. Furthermore, in order to put all variables ( $\boldsymbol{s}$ and $\boldsymbol{x}_{i p}$ ) on an equal footing, we set, for each variable, the mean to zero and standard deviation to unity (a linear conversion). This helps with the internal stability of most machine learning algorithms.

The free parameters, $\{w\}$, of the model are the learned error minimization using sets of data for which inputs and their corresponding outputs are known. This is an iterative procedure in which patterns are presented to the model, the outputs calculated, and the difference between these and the target outputs are used to perturb the weights in a direction that reduces the error. Learning is stopped once the rate of reduction of the error drops below some threshold. Our error function comprises two parts. The first term in the equation below is the sum-of-squares error in the predictions (the likelihood), the second is a regularization term,

$$
E=\sum_{p}\left(\frac{1}{2} \sum_{l} \beta_{l}\left[y(p)_{l}-T(p)_{l}\right]^{2}\right)+\alpha \frac{1}{2} \sum_{i} w_{i}^{2}
$$

where, for each pattern $p, T(p)_{l}$ and $y(p)_{l}$ are the target value and its estimate from the regression method for the $l$ th atmospheric parameter, respectively. The model (hyper) parameters $\beta_{l}$ dictate the relative importance of each parameter in the total error, and $\alpha$ specifies the degree of regularization. In the present work these hyperparameters were optimized via a brute force search (conditioned by experience). We actually use a "committee" of ten identical models trained on the same data, but trained from different initial random weights. Estimates of the atmospheric parameters obtained by the application of the model are the average of the ten individual estimates. This simple approach helps overcome "convergence" noise and, on average, increases the accuracy obtained.

Our estimate of the accuracy of the model in the application phase is the mean absolute error

$E=\frac{1}{P} \sum_{p=1}^{P}|C(p)-T(p)|$

where $C(p)$ is the committee estimate, and $T(p)$ is an independent estimate for the $p$ th spectrum. For the SR and RR models (see Sect. 5) $T$ is an estimate based on other methods (e.g., SSPP), so $E$ is not a real "error", but rather a discrepancy (as there is no definitive "ground truth").

\section{Dimensionality reduction}

Our initial models based on the full spectrum produced good results, but we find that the full spectrum is not necessary (not surprisingly, as it contains a large amount of redundant information). Dimensionality reduction often leads to enhanced reliability, because of the smaller number of parameters employed, and the considerably reduced computing time. We investigated various approaches and retained two - Principal Component 
Analysis (e.g.; Hastie et al. 2001; Singh et al. 2001; Bailer-Jones et al. 1998, and references therein) and a Wavelength Range Selection (e.g., Beers et al. 1999; Willemsen et al. 2005) - in the present work.

\subsection{Principal component analysis (PCA)}

Principal Component Analysis (PCA) linearly transforms a set of data via a rotation of the coordinate system, and an offset of its origin. The new axes (or principal components, the PCs) are chosen such that the projection of the data onto each axis in turn maximizes the variance in the data. If we have a set of $n$ vectors (spectra), $\boldsymbol{x}$, of dimension $N$ (the number of flux bins), then formally the principal components are the eigenvectors, $\boldsymbol{u}_{k}$ $(k=1 \ldots N)$, of the covariance matrix of the data. The $p$ th spectrum is reconstructed using the $\mathrm{PC}$ basis as

$\boldsymbol{y}_{p}(r)=\sum_{k=1}^{k=r} a_{k p} \boldsymbol{u}_{k}$

where

$a_{k p}=\boldsymbol{x}_{p} \cdot \boldsymbol{u}_{k}$

are the so-called "admixture coefficients". These represent the spectrum in the new (PC) space in the same way that the original spectrum did in the original (flux bin) space (i.e., they can be used as inputs in our regression models). If we set $r=N$ then we reconstruct the spectra exactly. If $r<N$ we have a reduced reconstruction, i.e., a compression which uses just the $r$ most significant PCs (those with the largest eigenvalues).

If the number of spectra is smaller than the dimensionality of the data, i.e., if $n<N$, then the spectra span a subspace of dimensionality $n$. In this case only $n$ PCs are defined and a full reconstruction is achieved with $N=n$. With $n \geq N$, then using all PCs in the reconstruction means that any spectrum - even one not used to form the PCs - can be reconstructed exactly. With $n<N$ this is no longer true. This is actually the case with our synthetic data, where $n=1816$ and $N=3818$. This potentially reduces the quality of any reconstruction, because some of the data space is not spanned by the PCs.

Reduced spectral reconstructions for five representative SDSS/SEGUE stars, using different numbers of eigenvectors computed from the synthetic and real spectra, are shown in Figs. 2 and 3 respectively. The residual spectrum, defined as the difference between the original and the reconstructed spectrum, is shown at the bottom for each pattern and each reconstruction. From inspection of these samples, one can see how the PCA approach acts as an effective filter to remove noise, recover missing and/or borderline features, and to detect outliers in a spectrum that are reconstructed with large errors (e.g., Storrie-Lombardi et al. 1995; Bailer-Jones et al. 1998). However, here we also note that there is evidence that the Kurucz model spectra we have adopted do not well describe SDSS/SEGUE spectra of cool stars $\left(T_{\text {eff }}<5000 \mathrm{~K}\right)$, especially when few PCs are retained in the reconstruction. The residual spectrum of main sequence stars having $T_{\text {eff }}=4431 \mathrm{~K}$ and $T_{\text {eff }}=4752 \mathrm{~K}$ highlights difficulties in reproducing, with $5+5$ and $25+25 \mathrm{PCs}$, the $\mathrm{C}_{2}$ band at $5165 \AA$ (see Fig. 2).

A useful measure of the reconstruction error over a set of $P$ spectra is

$Q(r)=\frac{1}{P}\left(\sum_{p=1}^{p=P} \frac{1}{N} \sum_{i=1}^{i=N}\left|\boldsymbol{x}_{i}-\boldsymbol{y}_{i}^{r}\right|\right)$.
Figure 4 shows how this error varies with $r$. Note that while the PCs themselves are constructed using the training data set, $Q(r)$ is calculated on a different set (namely the set to which the regression model is later applied). The three cases show quite different behaviour. For SS the error drops quite rapidly with increasing $r$, dropping to a constant (but non-zero) gradient after about 50 PCs (from a total of 1816), whereas for SR and RR the gradient of the curve becomes constant after including just a few PCs. The main reason is that real spectra (used either to form the PCs or in the projection) show much more variance than synthetic spectra, and this is spread over more data dimensions. A second observation is that the larger the noise, the larger the reconstruction error at a given $r$. For further discussion see Bailer-Jones (1996) or Bailer-Jones et al. (1998). It is interesting, however, that the curve for SR "levels off" at such a low value of $r$. This may well be a result of the fact, mentioned above, that the PCs only span a subspace of the original data space.

In summary, a PCA compression retains those spectral features which are most common across the data set. It preferentially removes noise (and rare features), because they are statistically uncorrelated. Note that the atmospheric parameters are not used in defining the PCs.

Thus, considering the above, the choice of the optimal number of PCs to retain is a trade-off between retaining information versus reducing dimensionality and noise, and should be optimized in conjunction with the regression model. There exist more sophisticated methods of dimensionality reduction which could be used in the future, such as local and nonlinear variations on PCA (see Einbeck et al. (2007) for a review and astronomical application).

\subsection{Wavelength range selection (WRS)}

The restriction of an analysis to certain wavelength intervals via the exclusion of (hopefully) unimportant ranges, is an alternative way to reduce the dimensionality of the input space. This provides a way of directly introducing domain information into the regression model. While this selection is potentially difficult (and the number of permutations extremely large), we show below that this approach is particularly effective for the estimation of the surface gravity parameter, $\log g$. After considering a number of alternatives, we chose to restrict the analysis on the wavelength ranges $3900-4400 \AA$, 4820-5000 ̊, 5155-5350 , and $8500-8700 \AA$ in the spectra. These regions contain the most prominent hydrogen and metal lines, including $\mathrm{CaII} \mathrm{K}$ and $\mathrm{H}$, the Balmer lines $\mathrm{H}_{\delta}, \mathrm{H}_{\gamma}$, and $\mathrm{H}_{\beta}$, the $\mathrm{CH}$ G-band, the $\mathrm{Mg} \mathrm{I} b$ triplet, and the CaII triplet.

\section{Results}

In this section we report the results of the three types of models developed, SS, RR and SR (for a definition of these see Sect. 1).

\subsection{SS - Synthetic vs. Synthetic}

For this analysis we adopt the sample of 1816 noise free synthetic spectra described in Sect. 2.2. This is randomly split into two equal-sized sets - one for model training, and one for model evaluation.

After a preliminary analysis with the full spectra, we decided to use a PCA pre-processing of the data (Sect. 4.1). Principal components are computed using the training set, then both sets are projected onto them to yield the admixture coefficients, 
P. Re Fiorentin et al.: Estimation of stellar atmospheric parameters from SDSS/SEGUE spectra
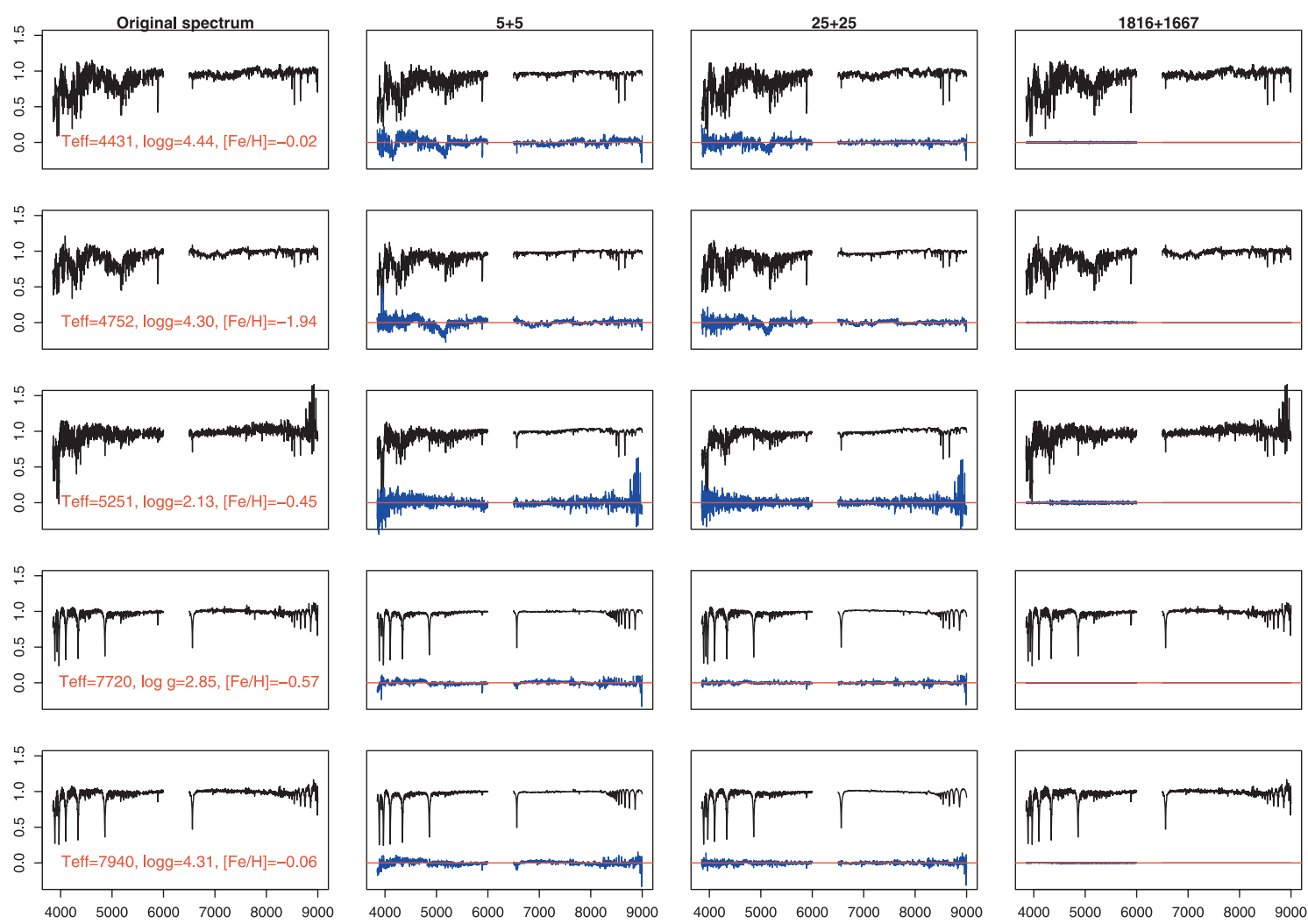

Fig. 2. Reconstruction of SDSS/SEGUE spectra by projection onto synthetic principal components. In each row, the spectrum on the left is the original and the following show the reconstruction using increasing numbers of principal components. The residual spectrum (original minus reconstructed) is shown in the bottom of each panel. The quoted atmospheric parameters are taken from a preliminary version of the processing pipeline SSPP.
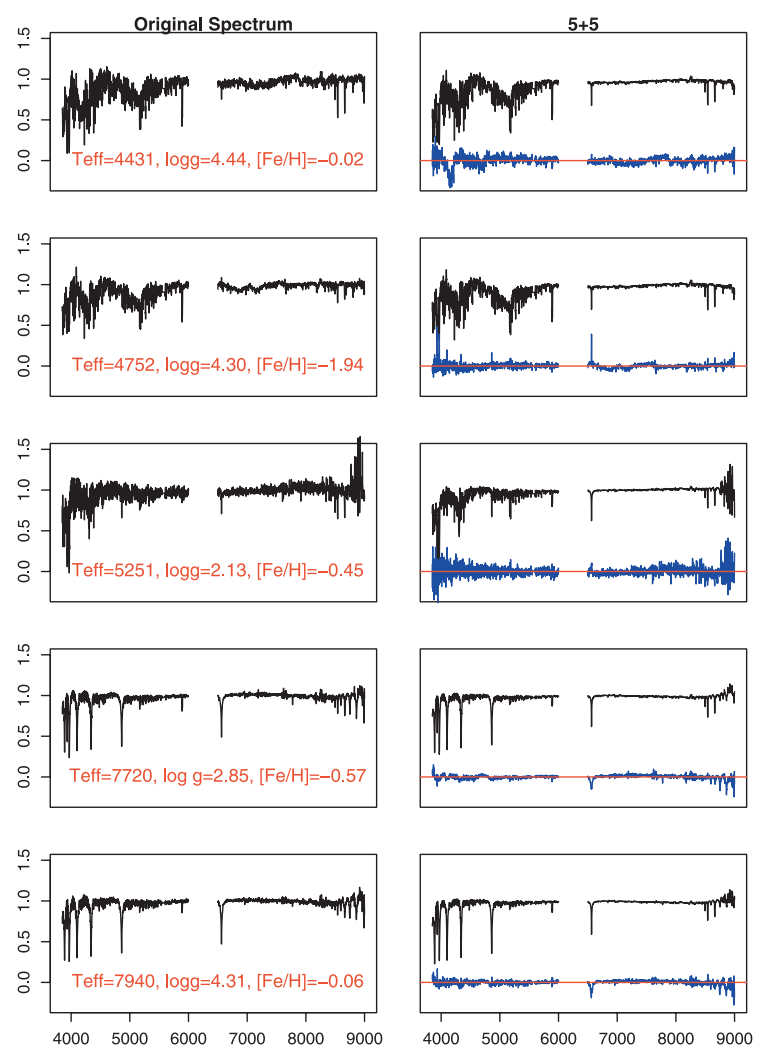
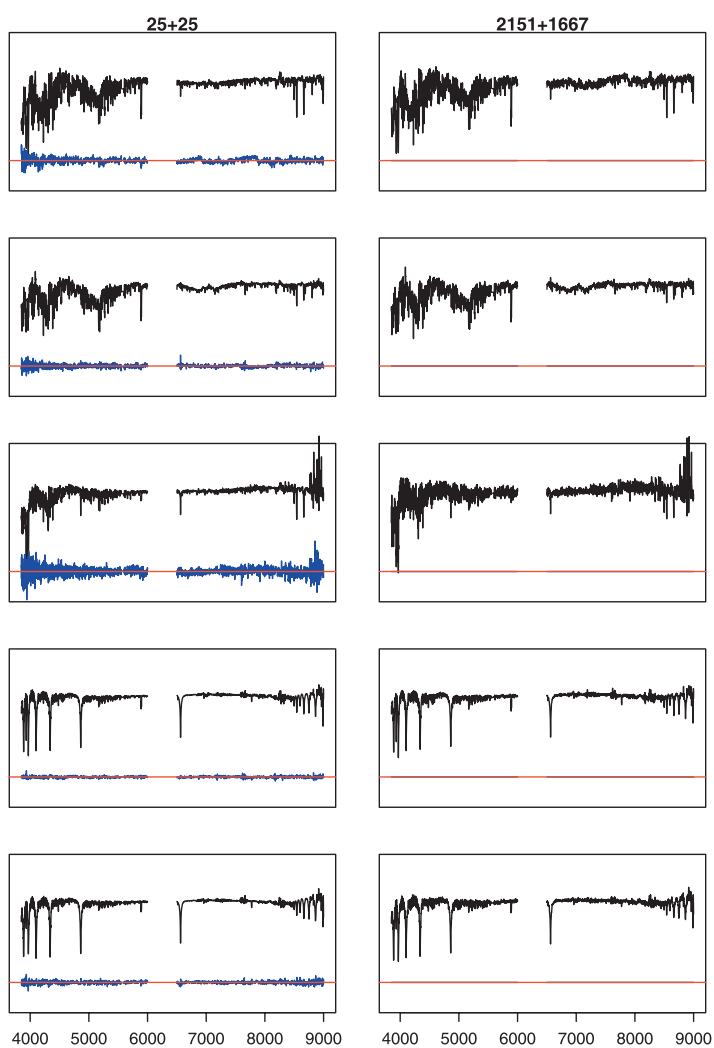

Fig. 3. As Fig. 2 but for principal components built from real spectra. 


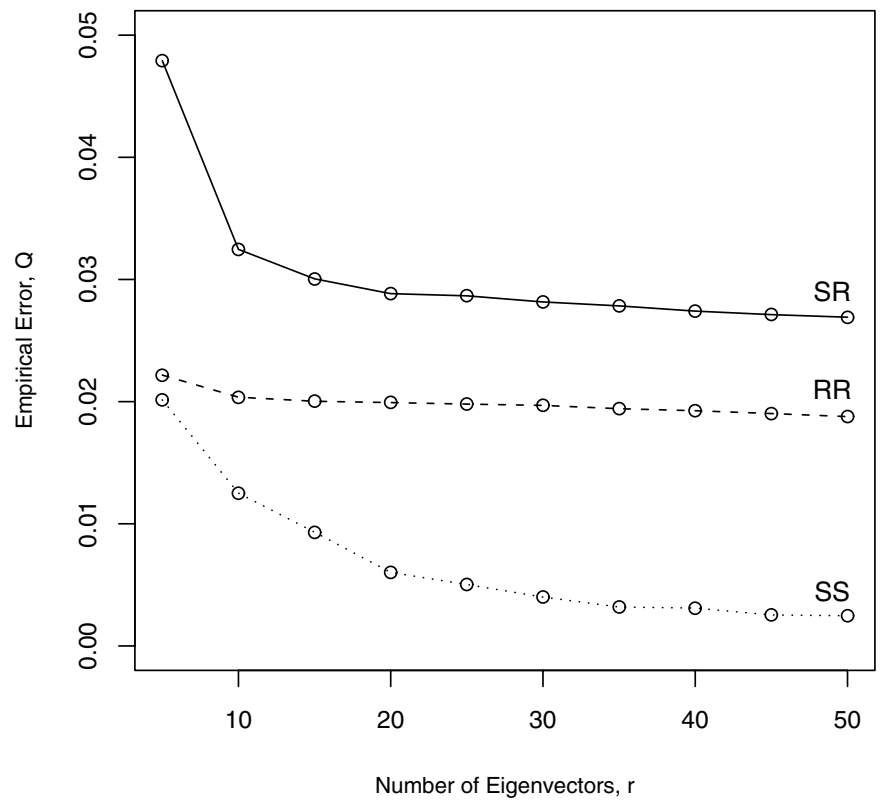

Fig. 4. PCA spectral reconstruction error, $Q$ (defined in Eq. 6) on the evaluation data set for SR/RR/SS (solid/dashed/dotted lines, respectively) as a function of the number of eigenvectors, $r$, used for reconstruction.

Table 1. Mean absolute errors on the evaluation set of 908 spectra in the SS model for different numbers of PCs retained in the reconstruction. (As PCA is done separately on the blue and red regions, the total number of inputs is twice the number of PCs.).

\begin{tabular}{llll}
\hline \hline PCs & $E_{\log T_{\text {eff }}}$ & $E_{\log g}$ & $E_{[\mathrm{Fe} / \mathrm{H}]}$ \\
\hline 5 & 0.0087 & 0.1264 & 0.1558 \\
25 & 0.0036 & 0.0245 & 0.0327 \\
100 & 0.0030 & 0.0251 & 0.0269 \\
908 & 0.0133 & 0.2087 & 0.2308 \\
\hline
\end{tabular}

which are then the regression model inputs. PCA is performed on the blue and red spectra separately, because this gave a better reconstruction (which in turn reduced systematic offsets in the derived parameters). Table 1 shows typical parametrization errors for the three stellar atmospheric parameters for different numbers of PCs retained in the reconstruction; they all are very small and surprisingly lower for $\log g$ than for $[\mathrm{Fe} / \mathrm{H}]$. We remark that, when increasing the number of PCs, the error is initially determined predominantly by the amount of information present in the reconstructed spectra, then by the limited ability of the non-linear regression model to make full use of the available information. These results, and the analysis of the reconstructed spectra, led us to select 25 (blue region) +25 (red region) PCs for the model.

The above results were obtained with noise-free data, which is not very realistic, so we also trained models where both the training and evaluation set are degraded with Gaussian additive noise to signal-to-noise (SNR) levels of 10/1, 30/1, 50/1 and $100 / 1$. Even at a $S N R$ of $10 / 1$, the errors are increased by only $50 \mathrm{~K}$ in $T_{\text {eff }}, 0.02$ dex in $\log g$, and $0.03 \mathrm{dex}$ in $[\mathrm{Fe} / \mathrm{H}]$. This modest deterioration is on account of the artifically good correspondence between the training and evaluation set when using purely synthetic data; the PCA noise filtering also appears to help. Note that whenever we use synthetic spectra to define the PCs, we always use noise-free spectra (also in Sect. 5.3).

\subsection{RR-Real vs. Real}

Following from our experience with the SS analysis, we build an RR regression model to parametrize real spectra. The training and evaluation data sets are taken from a set of 38731 stars from 140 SDSS/SEGUE plates, in directions of low reddening, which have had atmospheric parameters estimated by a preliminary version of the SSPP. Both training and evaluation sets are drawn at random (without replacement) with sizes 19731 and 19000 spectra respectively. We use 2151 pixels in the blue spectrum between $3850-6000 \AA$ and 1667 pixels in the red spectrum between $6500-9000 \AA$. A PCA compression reduces this to 25 (blue) +25 (red) PCs, the PCs themselves formed only from the training set. This compresses the data to $1.3 \%$ of its former size, resulting in more stable and faster models. We use these data to predict $\log T_{\text {eff }}, \log g$, and $[\mathrm{Fe} / \mathrm{H}]$. The standard deviations (essentially an estimate of their parameter ranges) of the input parameter distributions are $T_{\text {eff }}=724 \mathrm{~K}, \log g=0.64 \mathrm{dex}$, and $[\mathrm{Fe} / \mathrm{H}]=0.54 \mathrm{dex}$, respectively. These are on the order of the RMS errors which a random classifier would achieve.

In addition to this purely spectral model, we developed another model in which the four (de-reddened) photometric colours $u-g, g-r, r-i$, and $i-z$ are added as four additional model inputs (they are not involved in the PCA).

Figure 5 compares our model estimates with those from the SSPP on the evaluation set. Overall we see good consistency, especially for stars with $T_{\text {eff }}<8000 \mathrm{~K}\left(\log T_{\text {eff }}=3.90\right)$. Above this effective temperature we see that our models underestimate $\log T_{\text {eff }}$ relative to the SSPP. Our regression models are designed to smooth, i.e. interpolate, data. Extrapolation of the model to estimate atmospheric parameters that are not spanned by the training set is relatively unconstrained (and any model would need to make additional assumptions). Furthermore, the accuracy of the RR model is limited by the accuracy of the target atmospheric parameters used in training, as well as their consistency across the parameter space. In this case, the SSPP estimates are combinations from several estimation models, each of which operates only over a limited parameter range. Thus, the transition we see above $8000 \mathrm{~K}$ may indicate a temperature region where one of the SSPP submodels is dominating the SSPP estimates, and this is not well-generalized by our model. Of course, if we decided that we wanted to reproduce the SSPP predictions for hot stars, we could do this simply by fitting a second-order polynomial to our residuals to remove the systematic offset.

Table 2 quantifies the overall discrepacies for each parameter. An error in $\log T_{\text {eff }}$ of 0.0126 is an error of $2.9 \%$, or $170 \mathrm{~K}$ at $6000 \mathrm{~K}$. The last line in the table is the performance when we include photometry. Adding photometry leads to significant improvement in all three atmospheric parameters. This is not surprising for effective temperature, as the photometric calibration of these bands is less complicated than the spectral calibration. A more accurate $T_{\text {eff }}$ will permit more accurate $\log g$ and $[\mathrm{Fe} / \mathrm{H}]$. Thus, in directions where interstellar reddening is known to be low, photometry should be used. The values listed in the table for a given parameter are averaged over all values of the adopted atmospheric parameters. Results for gravity, metallicity, and effective temperature ranges - dwarfs/giants, low/high metallicity, and cool/warm stars - are listed in Table 5 and in Table 6.

\subsection{SR - Synthetic vs. Real}

We have shown above that our regression models are capable of obtaining accurate and consistent estimates of atmospheric parameters when trained and tested on synthetic spectra (SS), and 

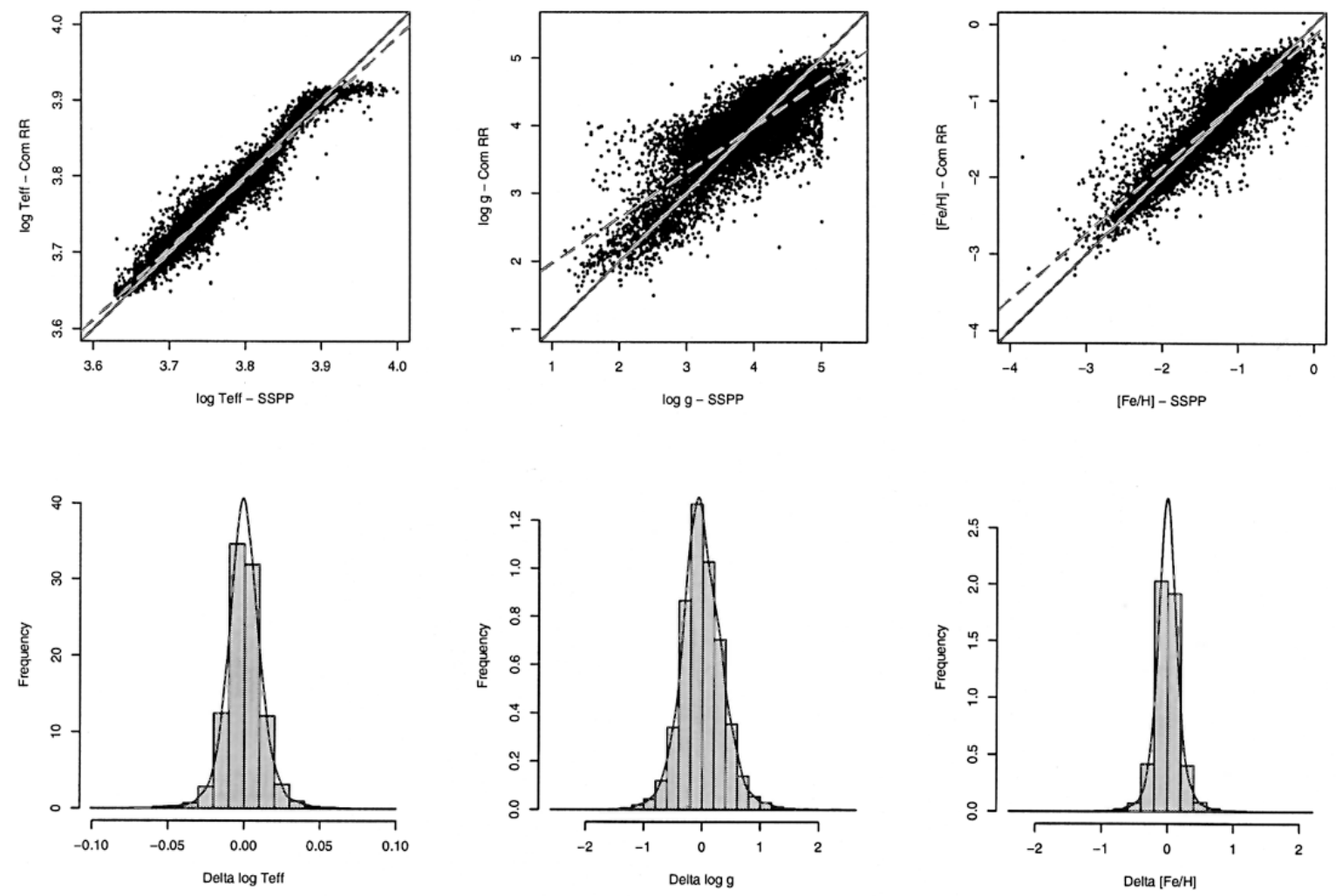

Fig. 5. Atmospheric parameters estimation with the RR model. We compare our estimated $\log T_{\text {eff }}, \log g$, and $[\mathrm{Fe} / \mathrm{H}]$ with those from a preliminary version of the SSPP on the 19000 stars in the evaluation set. The perfect correlation and a linear fit to the data are shown with the solid and dashed lines respectively. The histogram of the discrepancies (our estimates minus SSPP estimates) are shown in the lower panels.

Table 2. Mean absolute errors on the evaluation set of 19000 spectra in the RR model (plotted in Fig. 5). The first line is for the full data set (training and evaluation data). The second and third are just for the evaluation sets. The third line is for a model which included the four photometric colours as additional model inputs (predictors).

\begin{tabular}{llllll}
\hline \hline Set & PCs & + & $E_{\log T_{\text {eff }}}$ & $E_{\log g}$ & $E_{[\mathrm{Fe} / \mathrm{H}]}$ \\
\hline 38731 & $25+25$ & & 0.0090 & 0.2699 & 0.1339 \\
\hline 19000 & $25+25$ & & 0.0126 & 0.3644 & 0.1949 \\
& $25+25$ & phot & 0.0082 & 0.2791 & 0.1616 \\
\hline
\end{tabular}

also when trained on real spectra with existing parametrizations and applied to another sample of real spectra (RR). We now develop the hybrid approach, SR, in which we train on synthetic spectra and use this model to determine atmospheric parameters for SDSS/SEGUE spectra directly. A very important aspect of this model is processing the synthetic and real data to look similar; inaccurate synthetic spectra (e.g. poor models or a poor flux calibration) will degrade performance and/or give rise to systematic errors.

Experience shows that it is advantageous to match the noise properties of the synthetic training sample to that of the real sample. Essentially, noise acts as a regularizer in the training phase and thus improves the overall generalization performance of the models (e.g.; Snider et al. 2001; Odewahn et al. 2002), in particular reducing systematics. For each of the 38731 SDSS/SEGUE stars in the evaluation set we use the $S N R$ reported (for each pixel) in the data array included in the FITS file (which was estimated by the reduction pipeline). We assign a global $S N R$ to the spectrum which is the median of all flux bins over the wavelength range we retain (viz. 4000-5850 and 6500-8500 ).

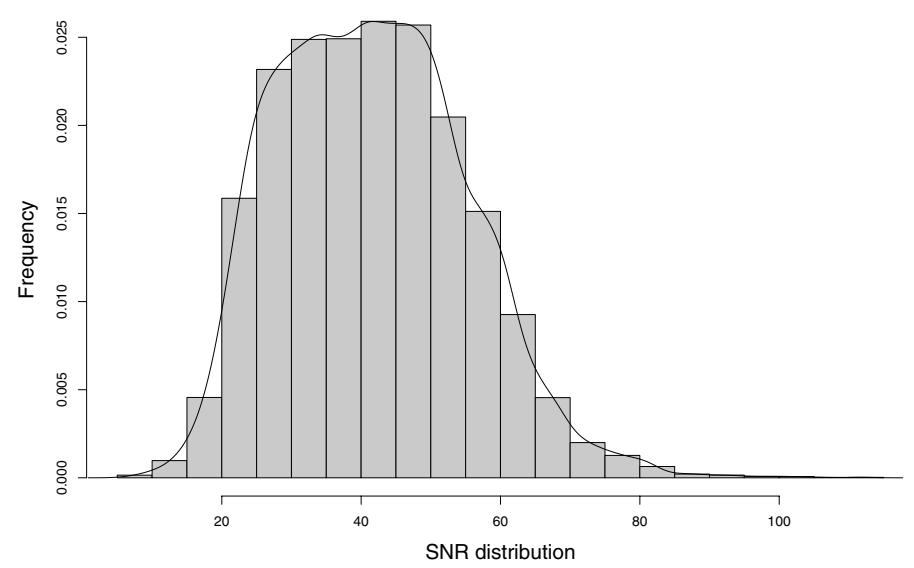

Fig. 6. Histogram of the $S N R$ distribution for all 38731 stars of the real sample. For each of them, the value for $S N R$ has been estimated from the stellar spectrum.

Figure 6 shows the distribution of these $S N R$ values. Based on this, we chose to develop two regression models, one optimized for low $S N R$ real spectra $(S N R<35 / 1,13487$ stars $)$ the other for high $S N R$ real spectra ( $S N R>35 / 1,25244$ stars). Experimentation showed that this noise injection does indeed reduce systematics which are obtained when using noise-free data for training.

We explored the application of dimensionality reduction with PCA, but found that this led to rather large systematic errors in the parameters, in particular in $\log g$ (up to $1.0 \mathrm{dex}$ ). We instead found that it is better simply to select wavelength regions which are known to be the most sensitive to surface gravity (e.g. 

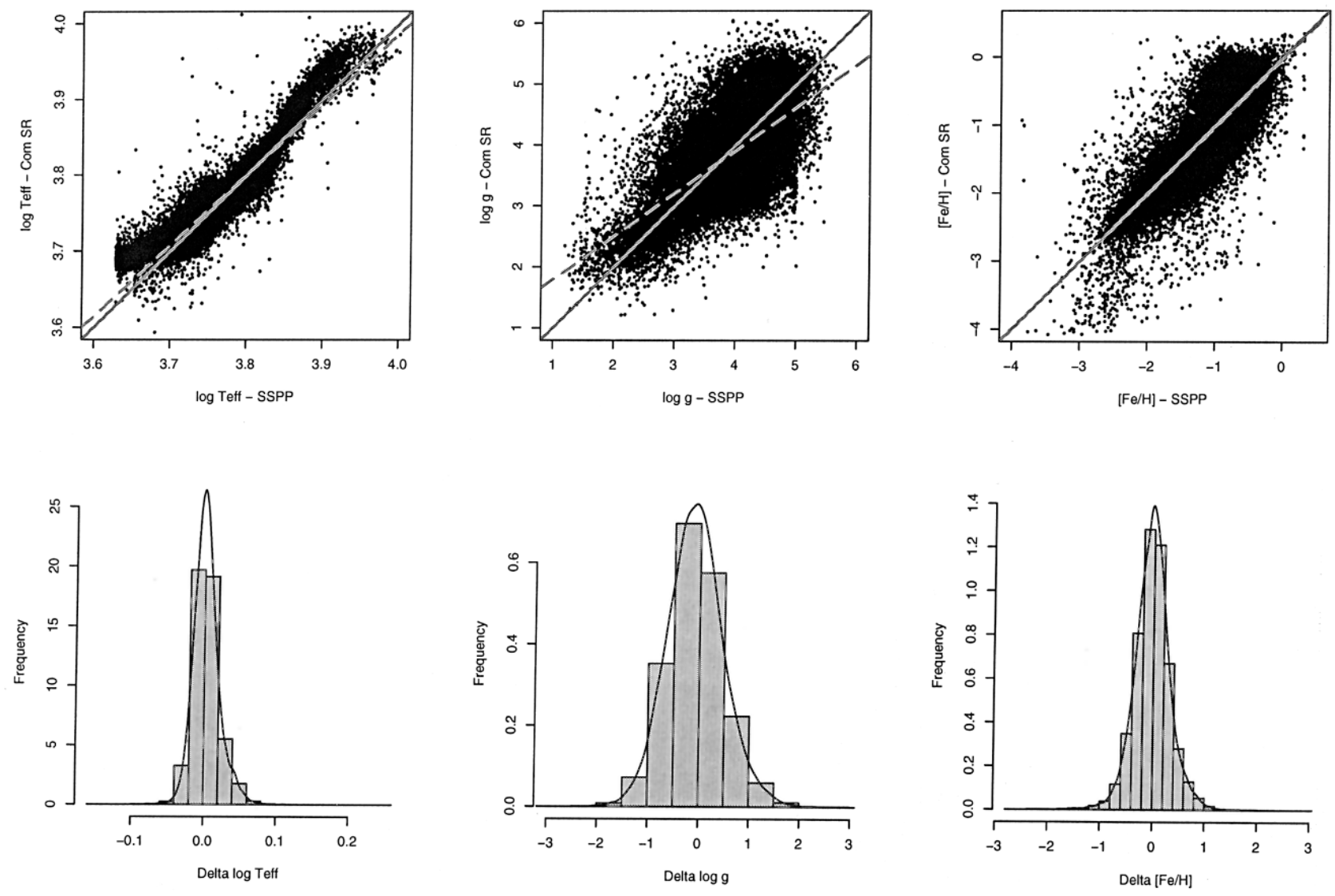

Fig. 7. Atmospheric parameters estimation with the SR model. Comparison between our derived $\log T_{\text {eff }}, \log g$, $[\mathrm{Fe} / \mathrm{H}]$ and those estimated by a preliminary version of SSPP for a set of 38731 stars. The perfect correlation and a linear fit to the data are shown with the solid and dashed lines respectively. The distribution of the residuals (model minus SSPP) are shown in the bottom panels.

$3900-4400 \AA$, $4820-5000 \AA, 5155-5350 \AA$ and $8500-8700 \AA)$. This is perhaps not unexpected, since essentially all of the methods that are used by the SSPP to define the target $\log g$ values use only these restricted wavelength ranges. This may also indicate that the gravity signature in real stars outside of the wavelength regions selected above behaves differently from the signature in the synthetic spectra. Either way, the excluded regions show less sensitivity to $\log g$, so for this parameter these regions do not add information, only data that are uncorrelated with the parameter of interest (so are effectively just noise). It is also possible, of course, that the PCA may be filtering out subtle (weak) features which are strong predictors of $\log g$.

Based on the above considerations, our final model uses PCA for estimating $T_{\text {eff }}$ and $[\mathrm{Fe} / \mathrm{H}]$ and WRS for estimating $\log g$. A separate model is used for estimating each parameter (although the $[\mathrm{Fe} / \mathrm{H}]$ model also predicts the other two, the results of which are disregarded).

Figure 7 compares our model atmospheric parameter estimates with those from the preliminary SSPP for the 38731 stars in the evaluation set. While the overall consistency between the two models is reasonably good, we (again) notice discrepancies at the extreme parameter values, in particular for $T_{\text {efff }}$. This is sometimes an indication that the model has not been well trained, i.e., it has not located a good local minimum of the error function (it can never be shown that the global minimum has been found with anything but an exhaustive search). However, there are inevitably problems with spectral mismatch, in the sense that the synthetic spectra do not reproduce all of the complexities of the spectra of real stars. The absence of
Table 3. Mean absolute discrepancies (between our SR model and SSPP) calculated on the evaluation set of 38731 real spectra (see also Fig. 7). Our models use PCA pre-processing for estimating $\log T_{\text {eff }}$ and $[\mathrm{Fe} / \mathrm{H}]$ and WRS pre-processing for estimating $\log g$; for the latter, PCA results are shown for comparison. Separate models were applied for low and high $S N R$ spectra (the transition being at $S N R=35 / 1$ ).

\begin{tabular}{lcccc}
\hline \hline Method & $S N R$ & $E_{\log T_{\text {eff }}}$ & $E_{\log g}$ & $E_{[\mathrm{Fe} / \mathrm{H}]}$ \\
\hline PCA (25+25) & & 0.0138 & 0.4288 & 0.2606 \\
& low & 0.0143 & 0.7549 & 0.3023 \\
& high & 0.0136 & 0.3465 & 0.2384 \\
\hline WRS & & - & 0.4459 & - \\
& low & - & 0.4495 & - \\
& high & - & 0.4450 & - \\
\hline
\end{tabular}

several molecular species in the linelists for the synthetic spectra may also be contributing to this problem, especially for cooler stars where they are expected to be more important. For the determination of metallicity, we observe that our model predicts lower metallicities for the lowest metallicity stars. This is probably a consequence of the lack of synthetic samples between $-4.0<[\mathrm{Fe} / \mathrm{H}]<-2.5$ (see Fig. 1) in our current grid.

Table 3 shows the global results (averaged over all stars and atmospheric parameters). It is interesting that the WRS preprocessing results in little difference in the $\log g$ discrepancy for the low and high $S N R$ regimes. Results for gravity, metallicity, and effective temperature ranges - dwarfs/giants, low/high metallicity, and cool/warm stars - are listed in Table 7 and in Table 8, and visualized in Fig. 8. We note that, in the estimation 

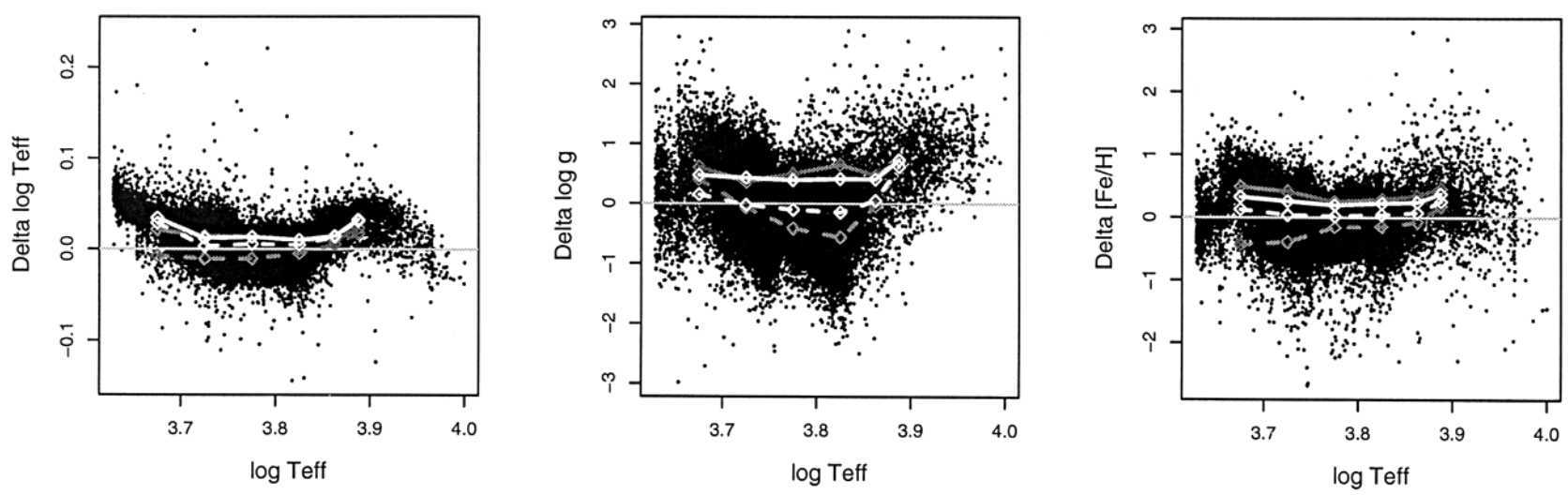

Fig. 8. More detailed visualization of the SR model discrepancies (Fig. 7). The diamonds joined by lines show mean absolute residual (solid lines) and mean residual (dashed lines) for low metallicity $([\mathrm{Fe} / \mathrm{H}]<-1.5$, white lines) and high metallicity $([\mathrm{Fe} / \mathrm{H}]>-1.5$, grey lines) averaged over all stars in a bin which has the diamond point as its centre. The mean residual traces the systematic offset (bias), the mean absolute the scatter.

of $\log g$, a systematic difference (our model predictions lower than SSPP) occurs in the range $T_{\text {eff }}=5600-6700 \mathrm{~K}$ for lowmetallicity giants. Unfortunately we cannot include photometry in the SR models, because the synthetic colours are not yet wellcalibrated, and their zero points on the $\mathrm{AB}$ system are still under discussion.

\subsection{Comparison of $R R$ and $S R$}

The RR and SR models developed above both appear to give reasonable predictions, as measured by their mean accuracies with respect to the SSPP predictions - $\log T_{\text {eff }}$ with residual of $0.013 / 0.014(\sim 170 \mathrm{~K}), \log g$ with a residual of $0.36 / 0.45 \mathrm{dex}$ and $[\mathrm{Fe} / \mathrm{H}]$ with a residual of $0.19 / 0.26$ dex for $\mathrm{RR} / \mathrm{SR}$ respectively.

The global discrepancies are larger with SR for $\log g$ and $[\mathrm{Fe} / \mathrm{H}]$, but this is not surprising because it is entirely independent of the SSPP parameter estimates. While the synthetic spectra place a limit on the performance of the SR model, this is true of any parametrization model. Physical parameters can only be derived using physical models; none can be measured "directly". The advantage of the SR approach is that it only uses one set in the parametrizations, it can easily be retrained using new synthetic spectra, and it provides a quick, general model which operates over the entire parameter range. In effect, the work in getting good predictions is taken out of the machine learning model and moved to the definition of the templates and the pre-processing.

We find that PCA delivers more accurate atmospheric parameters when the training data are the actual SDSS spectra with previously estimated parameters, whereas WRS appears superior for the estimation of $\log g$ templates, especially from lower SNR spectra.

From the subsample of 19000 stars used as the evaluation set in RR we compare the SR predictions with the RR predictions (see Fig. 9). The mean absolute differences are on the order of 0.010 in $\log T_{\text {eff }}(150 \mathrm{~K}), 0.35$ dex in $\log g$, and 0.22 dex in $[\mathrm{Fe} / \mathrm{H}]$.

\section{Application: globular clusters}

Comparison of theoretical isochrones with data from clusters offers an excellent opportunity to test the present model predictions. In particular, we can use them to assess the calibration of the parameter determinations. Here we focus our discussion on the globular cluster M 15, but we have also analysed the globular clusters M 13 and M 2 and the open cluster NGC 2420, all observed by SDSS/SEGUE. We select likely members, then estimate their atmospheric parameters, and overplot these on a set of isochrones fixed at literature values for the cluster distance modulus, age, and metallicity. If these values (and the isochrones themselves) are correct, discrepancies between our estimates and the isochrones would indicate problems in the calibrations of the atmospheric parameters (e.g. of the synthetic spectra on which the regression models are based). We note that Lee et al. (2007) have looked more carefully at the three globular clusters, and make an independent target selection based also on stellar densities, from which they derive mean metallicities and radial velocities for the clusters.

\subsection{15 - Selection}

The globular cluster M 15 is located in the sky at RA = $21^{\mathrm{h}} 29^{\mathrm{m}} 58.3^{\mathrm{s}}$, Dec $=+12^{\circ} 10^{\prime} 01^{\prime \prime}$ (Harris 1996), and has been extensively studied in the past (e.g., Sandage 1970; Binney \& Merrifield 1998). SDSS/SEGUE plates 1960 and 1962 include observations of its members. Figure 10 shows the distribution of the 526 stars with available SDSS/SEGUE spectroscopy and ugriz photometry. The central regions of the clusters are not generally available for spectroscopic observation, due to fibre placement restrictions in the SDSS spectrographs. This must be borne in mind when interpreting the results we describe below.

Based on position, we initially select 133 candidate members in the region $322^{\circ} .25<\mathrm{RA}<322^{\circ} .75$ and $11^{\circ} .90<\mathrm{Dec}<12^{\circ} .40$, as represented by the box shown in Fig. 10 .

The distribution of the atmospheric parameters $[\mathrm{Fe} / \mathrm{H}]$ versus log $g$ of this sample, using both the RR and SR models, is shown in Fig. 11. The stars clearly fall into two groups, due to false cluster members which we can plausibly take to be stars projected in front of the cluster from the Galactic field (generally at higher metallicity), and stars from the globular cluster itself (lower metallicity). It is also obvious that, given the apparent magnitude limits of SDSS/SEGUE, we would not expect to see higher-gravity main sequence stars that are true cluster members.

To obtain a more clean sample of likely cluster members, we select from the observed sample using published estimates of radial velocities and metallicities for the cluster (see Table 4).

We first select based on radial velocity; specifically, we retain as candidates only those stars with $-126 \mathrm{~km} \mathrm{~s}^{-1}<V_{R}<$ $-100 \mathrm{~km} \mathrm{~s}^{-1}$. This cut preferentially excludes metal-rich main 

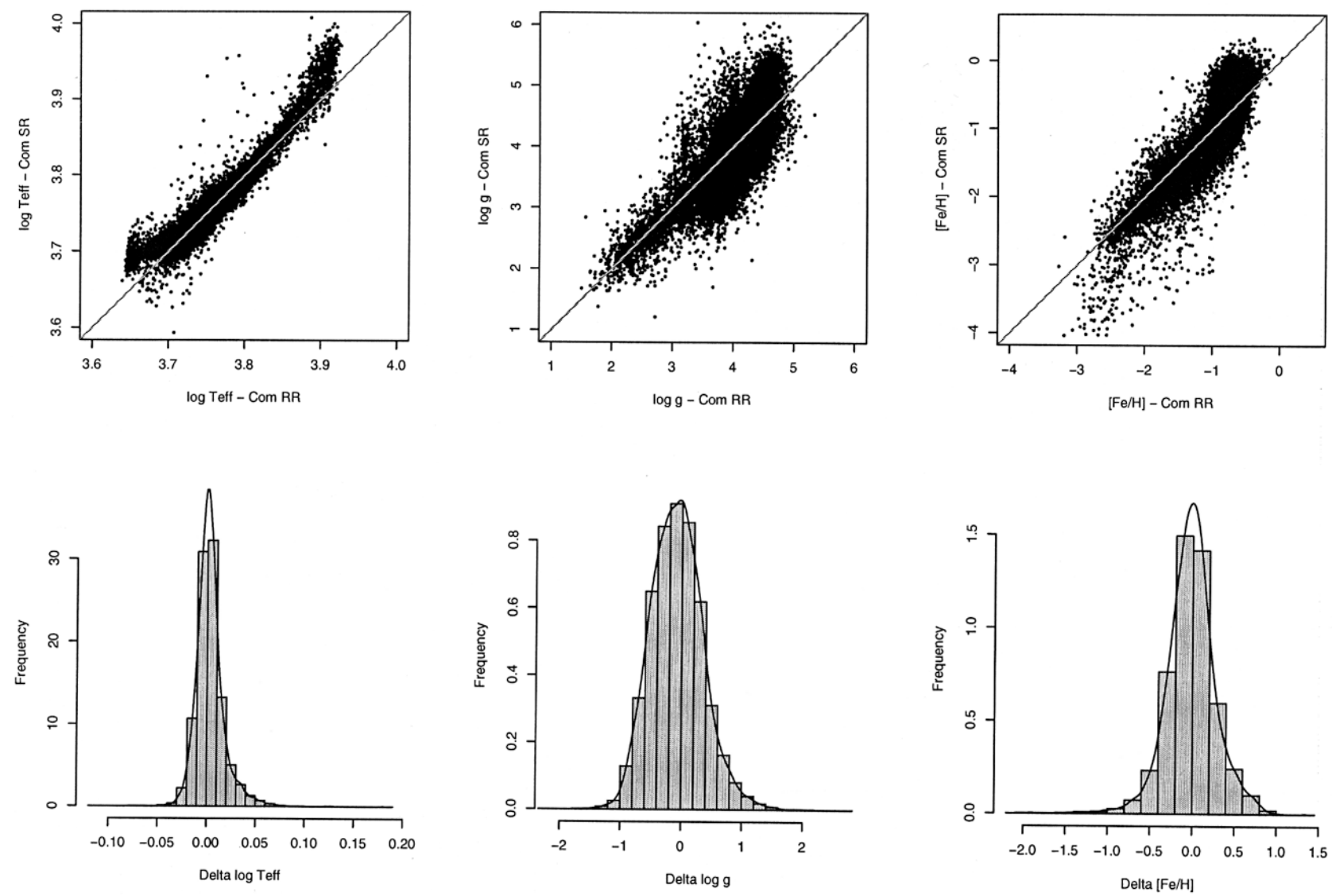

Fig. 9. Comparison between SR and RR estimations on the 19000 real spectra in common in their evaluation sets. The line shows the perfect correlation and the bottom panels the distributions of residuals.

sequence stars, and results in a remaining sample that contains 40 candidates with $[\mathrm{Fe} / \mathrm{H}]<-1.5$ out of a total of 42 .

We define a second sample, now of main sequence stars; namely, the 8 or 7 stars (for RR/SR respectively) having metal abundance $[\mathrm{Fe} / \mathrm{H}]<-1.5$ and $\log g>3.5$, without any radial velocity selection. Using the absolute magnitude determination for late-type dwarfs as a function of SDSS photometry (Bilir et al. 2005)

$M_{g}=5.791(g-r)+1.242(r-i)+1.412$

this second sample shows a distance modulus $(m-M)=14.67$, in agreement with the typical value $(m-M)_{\mathrm{M} 15}=14.93$ reported by Sandage (1970).

The complete sample of M 15 cluster members has 46 (RR)/ 45 (SR) stars. The entire radial velocity selected sample is shown in Fig. 11 with filled circles, while the metal-poor main sequence stars we suspect are cluster members are shown with asterisks.

M 15 has been previously analysed during the course of the development of the SSPP. Our initial sample (of 133 stars) includes 26 of the 35 candidates. Of these, 7 stars which have been rejected on the grounds of their apparently discrepant estimated abundance, or lack of an estimate at all, are marked with a plus sign. The 19 stars confirmed as likely members are also identified as part of our candidate members; we highlight these as grey dots in Fig. 11. Inspection of this figure shows the M 15 members as a clump of stars, albeit one which is more clumped in the RR predictions of the atmospheric parameters than in the SR predictions of the atmospheric parameters.

From the sample of cluster members with consistent metallicities and radial velocities we obtain a mean metallicity of
$[\mathrm{Fe} / \mathrm{H}]=-2.20 \pm 0.11 \mathrm{dex}(\mathrm{RR}) /[\mathrm{Fe} / \mathrm{H}]=-2.26 \pm 0.26 \mathrm{dex}$ (SR). Using just the giants in this sample (i.e., excluding the metal-poor main sequence stars) we obtain $[\mathrm{Fe} / \mathrm{H}]=-2.20 \pm$ $0.10 \mathrm{dex}(\mathrm{RR}) /[\mathrm{Fe} / \mathrm{H}]=-2.35 \pm 0.14 \mathrm{dex}(\mathrm{SR})$. These values are in good agreement with previous determinations in the literature (see Table 4).

\subsection{15 - Isochrones}

We now compare our atmospheric parameter estimates with theoretical SDSS isochrones from Girardi et al. (2004). We adopt an age of $13.2 \mathrm{Gyr}$, a metallicity $[\mathrm{Fe} / \mathrm{H}]=-2.22 \mathrm{dex}$, and a distance modulus of 14.93 (e.g., Sandage 1970; Binney \& Merrifield 1998).

Figure 12 shows the colour-magnitude and effective temperature-gravity diagrams for the likely M 15 members overplotted with the theoretical isochrones. These isochrones bracket the candidates reasonably well in the colour-magnitude diagram, but the distribution in the atmospheric parameter plane shows systematic offsets, in particular for the RR model estimates. A zero-point offset in either the gravity or temperature parameterizations (or in the isochrones) would improve the coincidence. On the other hand, the RR model clearly yields a tighter distribution in the atmospheric parameters. Thus, if we believe the isochrones, then we can conclude that the RR model obtains more precise parameter estimates, while the SR model obtains more accurate ones. In fact, if we would attribute the offset due entirely to gravity, we would have to apply corrections of about $0.60 \mathrm{dex}(\mathrm{RR})$ or $0.25 \mathrm{dex}(\mathrm{SR})$ to our estimates in 


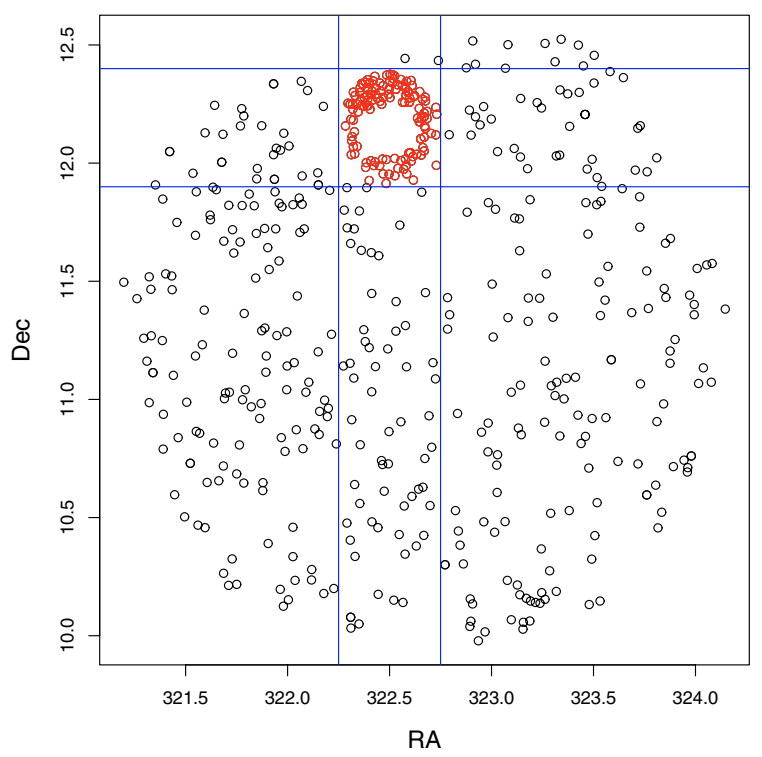

Fig. 10. Distribution on the sky of the 526 stars present from SDSS/SEGUE plates 1960 and 1962. The box defines the selection criteria $\left(322^{\circ} .25<\mathrm{RA}<322^{\circ} .75\right.$ and $11^{\circ} .90<$ Dec $\left.<12^{\circ} .40\right)$ which produces 133 M 15 candidates.
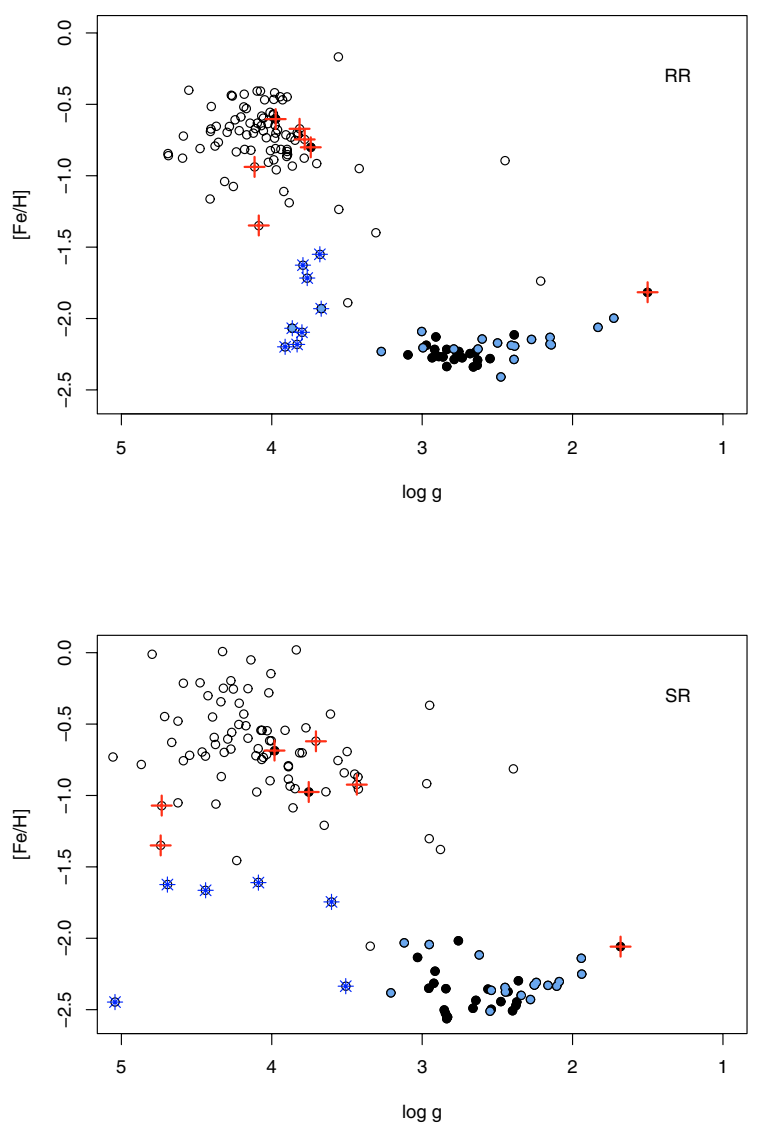

Fig. 11. Distribution of $[\mathrm{Fe} / \mathrm{H}]$ vs. $\log g$ for the 133 positionallyselected M 15 candidates from Fig. 10. Atmospheric parameters are from the RR (top) and SR (bottom) models. Of these 133 candidates, we retain only $46(\mathrm{RR})$ or 45 (SR) stars in the low-metallicity group as likely cluster members. Among these, 8 (RR) or 7 (SR) identified as main sequence stars (asterisks) and 40 by radial-velocity selection (filled dots). Those also selected as members in a preliminary analysis are highlighted in grey; members find to be doubtful (due to their measured abundances or lack of any metallicity estimate) are marked with a plus sign. order to obtain coincidence with their predicted location in the effective temperature-gravity planes.

\subsection{Other clusters}

We carried out the same analysis for three additional clusters which have also been extensively studied in the past, and so have reasonably consistent determinations of metallicity, age, and distance in the literature (see Table 4). Candidate stars from the globular clusters M 13 (e.g., Sandage 1970; Lupton et al. 1987; Shetrone 1994; Harris 1996; Binney \& Merrifield 1998) appear on SEGUE plates 2174, 2185, and 2255; from the globular cluster M 2 (e.g., Harris 1996; Lázaro et al. 2006) on SEGUE plate 1961; and from the open cluster NGC 2420 (e.g.; McClure et al. 1974; Smith 1987; Tianxing 1987) on SEGUE plates 2078 and 2079. For each of these, we select likely members following the same procedures as for the M 15 analysis (Sect. 6.1) and compare them with isochrones with parameters based on previous analyses.

Figure 13 shows the distribution of the atmospheric parameters for expected members of each cluster in the colourmagnitude and in the $\log T_{\text {eff }}-\log g$ plane, overplotted with the theoretical isochrones selected to best match each cluster's properties. Inspection of these distributions confirms our previous conclusions for the case of M 15 - (1) there exists a systematic offset in effective temperature and/or surface gravity between the estimated parameters and those expected from the theoretical isochrones, and (2) the RR model provides more precise atmospheric parameter estimates, while the SR model provides more accurate ones.

We are limited by the small number of likely cluster members in some cases, especially for M 2, which (so far) appears on only one SEGUE plate. However, it seems that this evidence is more clearly visible in the globular clusters which, as for M 15, are old and metal poor. In the atmospheric parameter plane, the distribution for the open cluster NGC 2420 from the SR model looks a bit confusing. It is plausible that this cluster is too metalrich to obtain good atmospheric parameter estimates, as the expected parameters are at the extreme of the regions covered by the synthetic grid used for training. Larger uncertainties are certainly present in this range of metallicity (see Tables 7, 8). These limitations are under study at the moment.

\section{Summary and conclusions}

We have developed models to estimate the three primary stellar atmospheric parameters $\left(T_{\text {eff }}, \log g\right.$, and $\left.[\mathrm{Fe} / \mathrm{H}]\right)$ from SDSS/SEGUE spectra. These models produce self-consistent parameter estimates and can be implemented into an automated data processing pipeline. Our models rely on an initial configuration (or "training") phase, which for one of the models (RR) uses pre-classified observed data, for the other (SR) synthetic spectra selected by the user. Both are flexible, in that new models can easily be introduced by changing the set of training templates.

Both models are nonlinear, regularized regression models. The RR model uses an initial PCA compression of the data to reduce the dimensionality (from 3818 to 50), thus producing a more robust (and precise) parametrizer (which reduces the dimensionality further to 3 , i.e., the three atmospheric parameters). They are also rapid, requiring of the order of one millisecond per star on a single, modest CPU.

The RR model has the advantage that exactly the same type of data are used in the training and application phases, thus 

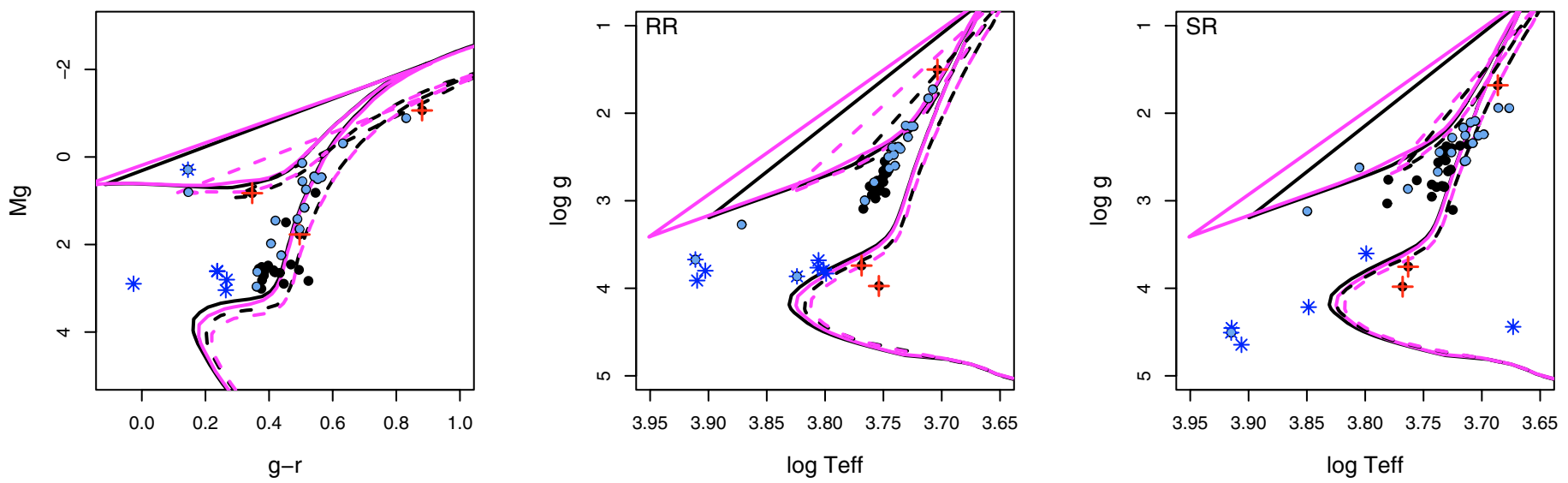

Fig. 12. The left panel shows the colour-magnitude diagram for M 15, and the two other panels the distribution of atmospheric parameters log $g$ vs. $\log T_{\text {eff }}$ from the RR (middle) and SR (right) models. Of the stars selected as candidates, the asterisks denote main sequence metal-poor stars, the filled dots the members based on radial velocity constrain. Confirmed and doubtful members assigned in a preliminary analysis are coloured grey and marked as plus sign respectively. Overplotted are isochrones from Girardi et al. (2004) with metallicities and ages which bracket the values given in Table 4, i.e. at $Z=0.0001$ (solid), $Z=0.0004$ (dashed) for ages of 12.59 Gyr (black) and 14.13 Gyr (grey).
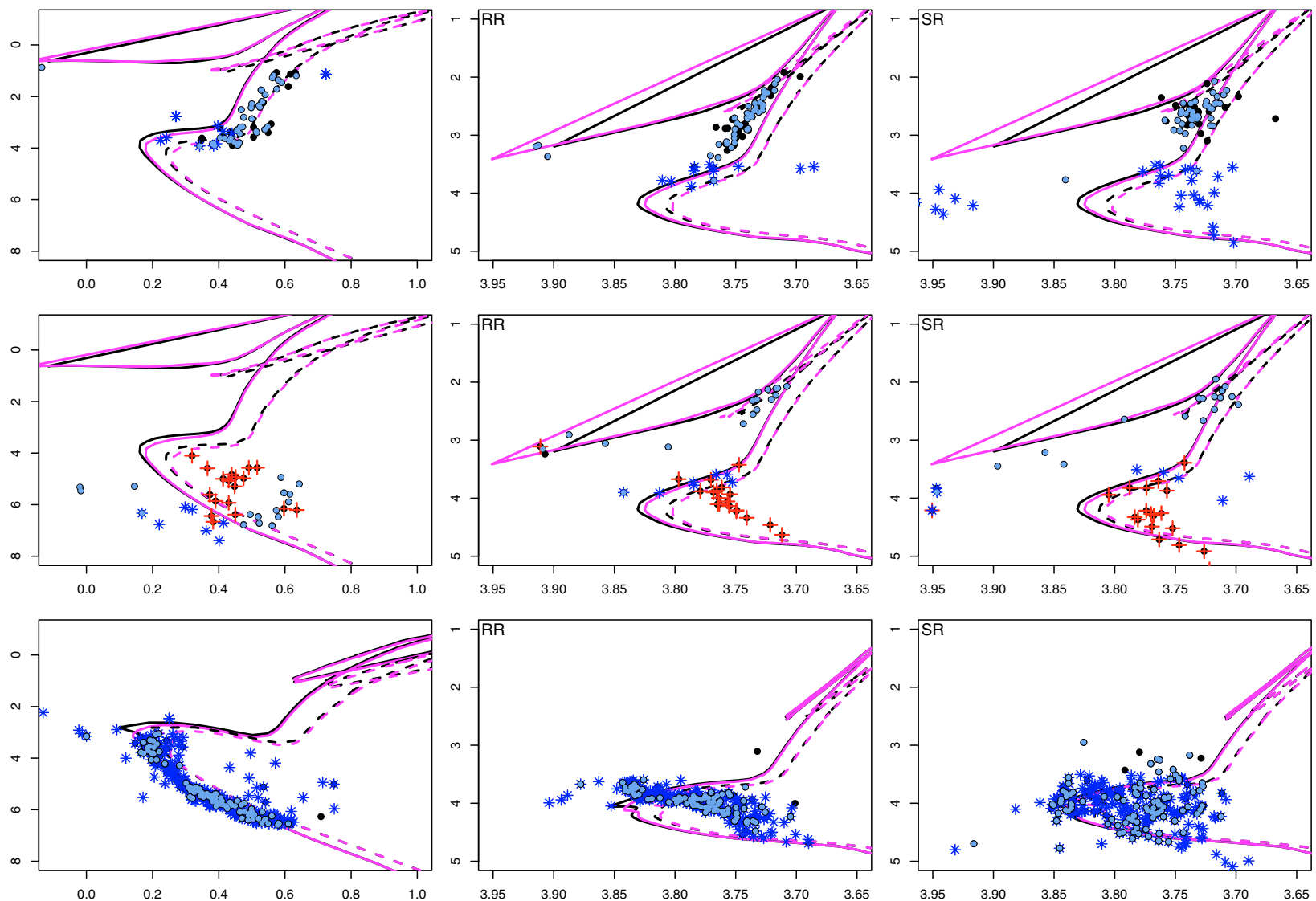

Fig. 13. As Fig. 12. Top: M 13 (globular cluster) candidates. Centre: M 2 (globular cluster) candidates. Bottom: NGC 2420 (open cluster) candidates. For the globular clusters, isochrones at $Z=0.0001$ (solid), $Z=0.0004$ (dashed) for ages of $12.59 \mathrm{Gyr}$ (black) and $14.13 \mathrm{Gyr}$ (grey); for the open cluster, isochrones at $Z=0.004$ (solid), $Z=0.008$ (dashed) for ages of $3.162 \mathrm{Gyr}$ (black) and $3.548 \mathrm{Gyr}$ (grey).

Table 4. Globular/Open Clusters, literature values. The selection constraints applied for identification of likely members are labeled with $*$.

\begin{tabular}{lccccccrr}
\hline \hline Cluster & RA, Dec (epoch J2000) & $\begin{array}{c}\text { RA }^{*} \\
(\text { degree })\end{array}$ & $\begin{array}{c}\text { Dec } \\
(\text { degree })\end{array}$ & $\begin{array}{c}{[\mathrm{Fe} / \mathrm{H}]} \\
(\mathrm{dex})\end{array}$ & $\begin{array}{c}\text { age } \\
(\mathrm{Gyr})\end{array}$ & $m-M$ & $\begin{array}{r}R V \\
\left(\mathrm{~km} \mathrm{~s}^{-1}\right)\end{array}$ & $\begin{array}{r}R V^{*} \\
\left(\mathrm{~km} \mathrm{~s}^{-1}\right)\end{array}$ \\
\hline M 15 & $21^{\mathrm{h}} 29^{\mathrm{m}} 58.3^{\mathrm{s}},+12^{\circ} 10^{\prime} 01^{\prime \prime}$ & $322.25,322.75$ & $11.90,12.40$ & -2.22 & 13.2 & 14.93 & -110 & $-126,-100$ \\
M 13 & $16^{\mathrm{h}} 41^{\mathrm{m}} 41.5^{\mathrm{s}},+36^{\circ} 27^{\prime} 37^{\prime \prime}$ & $250.00,250.90$ & $36.10,36.90$ & -1.70 & 12.7 & 14.07 & -250 & $-262,-243$ \\
M 2 & $21^{\mathrm{h}} 33^{\mathrm{m}} 29.3^{\mathrm{s}},-00^{\circ} 49^{\prime} 23^{\prime \prime}$ & $323.10,323.60$ & $-1.05,-0.60$ & -1.53 & 13.0 & 10.49 & 0 & $-20,20$ \\
NGC 2420 & $07^{\mathrm{h}} 38^{\mathrm{m}} 24.0^{\mathrm{s}},+21^{\circ} 34^{\prime} 27^{\prime \prime}$ & $114.40,115.10$ & $21.20,22.10$ & -0.50 & $3 / 4$ & 11.40 & 73 & 50,86 \\
\hline
\end{tabular}


Table 5. RR: partial results. We list the mean $\mu$ and the corresponding standard deviation $\sigma$ of the difference Committee-SSPP for each of the different stellar types and parameter ranges.

\begin{tabular}{|c|c|c|c|c|c|c|c|c|c|c|}
\hline$\overline{[\mathrm{Fe} / \mathrm{H}]}$ & $\log T_{\text {eff }}$ & & $\log _{\mathrm{e}} \mathrm{eff}$ & $E_{\log T_{\mathrm{eff}}}$ & $\mu_{\log g}$ & $\sigma_{\log g}$ & $\overline{E_{\log g}}$ & $\mu_{[\mathrm{Fe} / \mathrm{H}]}$ & $\sigma_{[\mathrm{Fe} / \mathrm{H}]}$ & $\overline{E_{[\mathrm{Fe} / \mathrm{H}]}}$ \\
\hline$<-1.5$ & $<3.70$ & 0.0155 & 0.0166 & 0.0172 & 0.1879 & 0.4448 & 0.3724 & 0.2673 & 0.2733 & 0.3056 \\
\hline$<-1.5$ & $3.70,3.75$ & 0.0061 & 34 & 0.0105 & 0.1214 & 939 & 0.3075 & 817 & 2072 & 0.1464 \\
\hline$<-1.5$ & $3.75,3.80$ & 0.0011 & 0.0097 & 0.0073 & 0.1284 & 0.3463 & 0.2823 & 0.0619 & 531 & 0.1266 \\
\hline$<-1.5$ & $3.80,3.85$ & -0.0032 & 0.0107 & 0.0082 & -0.1141 & 0.4439 & 0.3606 & & 2048 & 0.1484 \\
\hline$<-1.5$ & $3.85,3.875$ & 46 & & 0. & & & 0.4117 & & 337 & 0.2882 \\
\hline$<-1.5$ & $>3.875$ & -0.0044 & 30 & 0.0173 & 0.0007 & 0.3487 & 0.2412 & 0.2860 & 164 & 0.3875 \\
\hline$>-1.5$ & $<3.70$ & 0.0087 & & 8 & 0.0941 & 0.3696 & 0.2882 & 0.0295 & 150 & 0.1658 \\
\hline$>-1.5$ & $70,3.75$ & & & & & & & & & 1044 \\
\hline$>-1.5$ & $3.75,3$ & -0.0 & & & 55 & & & 59 & & 139 \\
\hline$>-1.5$ & 0,3 & -0.1 & & & 0 & & & 3 & & 904 \\
\hline$>-1.5$ & $3.85,3.875$ & & & & -0. & & & 4 & & 898 \\
\hline$>-1.5$ & $>3.875$ & -0.0119 & 0.0189 & 0.0151 & -0.0361 & 0.4226 & 0.3085 & -0.2144 & 0.3799 & 0.3226 \\
\hline $\log g$ & $\overline{\log T_{\text {eff }}}$ & & & & & & & & & \\
\hline$<3.5$ & $<3.70$ & 93 & 164 & 0.4302 & 0.3427 & 0.4407 & 0.4302 & 0.1685 & 0.2540 & 0.2347 \\
\hline$<3.5$ & $3.70,3.75$ & & & & & & 0.3577 & & & 0.1454 \\
\hline$<3.5$ & $3.75,3.80$ & & & & & & 0.4115 & & & 0.1401 \\
\hline$<3.5$ & $3.80,3.85$ & & & & & & 0.6220 & & & 0.1946 \\
\hline$<3.5$ & $3.85,3.875$ & & & & & & 0.5691 & & & 0.2347 \\
\hline$<3.5$ & $>3.875$ & -0.0078 & 0.0240 & 0.2639 & 0.1860 & 0.3 & 0.2639 & 0.0662 & 0.4635 & 0.3435 \\
\hline$>3.5$ & $<3.70$ & 0.0069 & & 0.0105 & & & 0.2644 & & 0.2226 & 0.1692 \\
\hline$>3.5$ & $70,3.75$ & 0.0001 & & 0075 & -0.0207 & & 0.2216 & & & 0.0968 \\
\hline$>3.5$ & $3.75,3.80$ & -0.0035 & & 0.0080 & -0.1172 & & 0.2188 & -0.0300 & & 0.1116 \\
\hline$>3.5$ & $3.80,3.85$ & -0.0054 & & & & & & & & 0.1717 \\
\hline$>3.5$ & $3.85,3.875$ & & & & $\delta 8$ & & 497 & 00 & & 0.2005 \\
\hline$>3.5$ & $>3.875$ & -0.0092 & 0.0190 & 0.0146 & -0.1452 & 0.3797 & 0.2874 & -0.0367 & 0.4618 & 0.3521 \\
\hline
\end{tabular}

Table 6. RR: partial results. We list the mean $\mu$ and the corresponding standard deviation $\sigma$ of the difference Committee-SSPP for each of the different stellar temperatures and metallicity ranges.

\begin{tabular}{ccrrc}
\hline \hline$T_{\text {eff }}$ & {$[\mathrm{Fe} / \mathrm{H}]$} & $\mu_{[\mathrm{Fe} / \mathrm{H}]}$ & $\sigma_{[\mathrm{Fe} / \mathrm{H}]}$ & $E_{[\mathrm{Fe} / \mathrm{H}]}$ \\
\hline$<4500$ & $<-2.5$ & 0.9062 & 0.8550 & 0.9062 \\
$<4500$ & $-2.5,-2.0$ & 0.2322 & 0.1414 & 0.2322 \\
$<4500$ & $-2.0,-1.5$ & 0.3464 & 0.3664 & 0.4503 \\
$<4500$ & $-1.5,-1.0$ & 0.1196 & 0.1779 & 0.1667 \\
$<4500$ & $-1.0,-0.5$ & -0.1419 & 0.1634 & 0.1732 \\
$<4500$ & $>-0.5$ & -0.4616 & 0.1322 & 0.4616 \\
\hline 4500,6500 & $<-2.5$ & 0.1183 & 0.1770 & 0.1574 \\
4500,6500 & $-2.5,-2.0$ & -0.0043 & 0.1608 & 0.1144 \\
4500,6500 & $-2.0,-1.5$ & -0.0133 & 0.1636 & 0.1219 \\
4500,6500 & $-1.5,-1.0$ & -0.0435 & 0.1683 & 0.1303 \\
4500,6500 & $-1.0,-0.5$ & -0.0294 & 0.1291 & 0.0999 \\
4500,6500 & $>-0.5$ & -0.1416 & 0.1207 & 0.1456 \\
\hline
\end{tabular}

eliminating the issue of discrepancies in the flux calibration or cosmic variance of the two samples. Of course, this requires an independent estimation method ("basis parameterizer") to parametrize the training templates (which itself must use synthetic models at some level). Our regression model then automates and - more importantly - generalizes this basis parameterizer. Indeed, the basis parameterizer may even comprise multiple algorithms, perhaps operating over different parameters ranges or used in a voting system to estimate atmospheric parameters. This is true in the present case, where the basis parameterizer comes from a preliminary version of the SDSS/SEGUE Spectroscopic Parameter Pipeline (SSPP; Beers et al. 2006; Lee et al. 2007).

In contrast, our SR model is trained directly on synthetic spectra, dispensing with the need for a basis parameterizer. For best results these training data should have noise properties similar to the observed data (which improves the regularization). We therefore implemented different models for different SNR ranges. PCA is again used for data compression, except for the surface gravity parameter log $g$, where better results were obtained using a subset of spectral features known to be most sensitive to this parameter.

For each atmospheric parameter, the accuracy of our predictions with respect to previous estimates (SSPP) are $T_{\text {eff }}$ to $170 / 170 \mathrm{~K}, \log g$ to $0.36 / 0.45 \mathrm{dex}$ and $[\mathrm{Fe} / \mathrm{H}]$ to $0.19 / 0.26 \mathrm{dex}$ for methods RR and SR respectively. Consistency between the two approaches is on order of $150 \mathrm{~K}$ in $T_{\text {eff }}, 0.35 \mathrm{dex}$ in $\log g$, and 0.22 dex in $[\mathrm{Fe} / \mathrm{H}]$. Some discrepancies are probably due to the different Kurucz models adopted in our SR model and in some of the methods employed in the SSPP.

As a test of our model predictions, we estimated atmospheric parameters for globular/open cluster members and compared these to theoretical isochrones. We found that RR gives more precise parameter estimates (stars show smaller scatter) whereas SR gives more accurate ones (stars show smaller offset, or bias). We can use this information to improve the parameter calibration of the basis parametrizers or the pre-processing of the synthetic spectra. We have also used our models to estimate atmospheric parameters for 89600 SEGUE and 194172 SDSS (DR-5) stellar spectra, which are being used for further scientific investigations.

We found that the inclusion of the four SDSS photometric colours improves the precision of parameter estimation significantly, but this will only work for zero (or very low) extinction regions. In principle, our models can be extended to predict extinction (by inclusion of its variance in the training set), allowing us to then use both photometry and spectroscopy to predict atmospheric parameters along significantly reddened lines of sight.

Our RR model has already been successfully integrated into the SSPP. The SR will undergo further refinement with improved synthetic spectra. In particular, models with more molecules 
Table 7. SR: partial results. We list the mean $\mu$ and the corresponding standard deviation $\sigma$ of the difference Committee-SSPP for each of the different stellar types and parameter ranges.

\begin{tabular}{|c|c|c|c|c|c|c|c|c|c|c|}
\hline$[\mathrm{Fe} / \mathrm{H}]$ & $\bar{l} \log T_{\text {eff }}$ & $\mu_{\log }$ & $\sigma_{\log }$ & $\overline{E E_{\log }}$ & $\mu_{\log }$ & $\overline{\overline{\sigma_{\text {log }}}}$ & $\overline{E_{\log }}$ & $\mu_{[\mathrm{Fe} / \mathrm{H}]}$ & $\bar{\sigma}_{[\mathrm{Fe} / \mathrm{H}]}$ & $E_{[\mathrm{Fe} / \mathrm{H}]}$ \\
\hline$<-1.5$ & $<3.70$ & -0.0076 & 0.0272 & 0.0212 & 0.4070 & 0.6537 & 0.6097 & -0.4354 & 0.4470 & 0.4902 \\
\hline$<-1.5$ & $3.70,3.75$ & -0.0105 & 0.0143 & 0.0143 & -0.0665 & 0.4660 & 0.3627 & -0.3936 & 0.3146 & 0.4099 \\
\hline$<-1.5$ & $3.75,3.80$ & -0 & 0.0129 & 0.0133 & -0.4136 & 0.4600 & 0.5013 & -0 . & 294 & 0.2454 \\
\hline$<-1.5$ & $3.80,3.85$ & -0.0047 & 0.0117 & 0.0098 & -0.5609 & 5208 & 0.6488 & -0 . & 91 & 0.2873 \\
\hline$<-1.5$ & $3.85,3.875$ & 0.0051 & 0.0147 & 0.0099 & -0.0387 & 0.6575 & 0.4753 & -0.0771 & 16 & 0.3410 \\
\hline$<-1.5$ & $>3.875$ & 0.0171 & 0.0230 & 0.0228 & 0.6129 & 0.5321 & 0.6973 & 750 & 35 & 0.4622 \\
\hline$>-1.5$ & $<3.70$ & 88 & .0254 & $\overline{38}$ & 0.1509 & 0.5730 & 0.4750 & $\overline{275}$ & 51 & 0.3267 \\
\hline$>-1.5$ & $3.70,3.75$ & 30 & 0.0 & 12 & -0.0213 & 0.5409 & .4298 & 76 & 22 & 0.2593 \\
\hline$>-1.5$ & $3.75,3.80$ & 0.0054 & 0.0 & 0.0 & -0.1007 & 0.4923 & 0.3937 & 76 & 45 & 0.1955 \\
\hline$>-1.5$ & $3.80,3.85$ & 0.0045 & 0.0140 & 0.0099 & -0.1443 & 0.5179 & 0.4154 & 415 & 76 & 0.2261 \\
\hline$>-1.5$ & $3.85,3.875$ & 0.0136 & 0.0119 & 0.0142 & 0.0482 & 0.5324 & 0.4087 & 744 & 325 & 0.2408 \\
\hline$>-1.5$ & $>3.875$ & 0.0310 & 0.0200 & 0.0320 & 0.6196 & 0.6062 & 0.7288 & 0.2546 & 0.4465 & 0.3759 \\
\hline $\log g$ & $\log T_{\text {eff }}$ & & $\sigma_{\mathrm{l}}$ & $E_{\mathrm{lo}}$ & $\mu_{\log }$ & $\sigma$ & $E_{\mathrm{l}}$ & $\mu$ & $\sigma_{-}$ & $E_{[\mathrm{Fe} / \mathrm{H}]}$ \\
\hline 3.5 & $<3.70$ & -0.0099 & 0.0278 & 0.4282 & 0.0085 & 0.5627 & 0.4282 & -0.3130 & 0.4795 & 0.3967 \\
\hline 3.5 & $3.70,3.75$ & -0.0065 & 0.0 & 0. & -0.2002 & 0.4827 & 0.4088 & -0 . & 19 & 0.3121 \\
\hline 3.5 & $3.75,3.80$ & -0.0068 & 0.0160 & 0.5700 & -0.5161 & 0.4468 & 0.5700 & -0.1164 & 367 & 0.2257 \\
\hline 3.5 & $3.80,3.85$ & -0.0015 & 0.0127 & & -0.6085 & 0.5020 & 0.6804 & -0 . & & 0.2766 \\
\hline 3.5 & $3.85,3.875$ & & & & -0.2528 & 0.6760 & 0.5740 & 592 & & 0.3449 \\
\hline 3.5 & $>3.875$ & & 0.0290 & 0.5496 & 0.1515 & 0.6719 & 0.5496 & 883 & 792 & 0.4839 \\
\hline 3.5 & $<3.70$ & & 0.0275 & & 0.3073 & 0.6112 & 0.5486 & 49 & 90 & 0.3772 \\
\hline 3.5 & $70,3.75$ & & 0.0167 & & 0.0297 & 0.5323 & 0.4223 & & & 0.2741 \\
\hline 3.5 & $75,3.80$ & & 0.0156 & & 0.0039 & 0.4320 & 0.3393 & & & 0.1987 \\
\hline 3.5 & $0,3.85$ & & 0.0144 & & -0.0841 & 0.4873 & 0.3789 & & & 0.2275 \\
\hline 3.5 & $85,3.875$ & & 0.0117 & & 0.0866 & 0.5113 & 0.3907 & 520 & 288 & 0.2391 \\
\hline 3.5 & $>3.875$ & 0.0272 & 0.0207 & 0.0297 & 0.6556 & 0.5666 & 0.7354 & 0.2241 & 0.4747 & 0.3885 \\
\hline
\end{tabular}

Table 8. SR: Partial results. We list the mean $\mu$ and the corresponding standard deviation $\sigma$ of the difference Committee-SSPP for each of the different stellar temperatures and metallicity ranges.

\begin{tabular}{ccrrr}
\hline \hline$T_{\text {eff }}$ & {$[\mathrm{Fe} / \mathrm{H}]$} & $\mu_{[\mathrm{Fe} / \mathrm{H}]}$ & $\sigma_{[\mathrm{Fe} / \mathrm{H}]}$ & $E_{[\mathrm{Fe} / \mathrm{H}]}$ \\
\hline$<4500$ & $<-2.5$ & -0.9158 & 0.6332 & 0.9158 \\
$<4500$ & $-2.5,-2.0$ & -0.2961 & 0.4686 & 0.4342 \\
$<4500$ & $-2.0,-1.5$ & 0.2013 & 0.6026 & 0.4545 \\
$<4500$ & $-1.5,-1.0$ & 0.2022 & 0.4808 & 0.3597 \\
$<4500$ & $-1.0,-0.5$ & -0.4289 & - & 0.4289 \\
$<4500$ & $>-0.5$ & - & - & - \\
\hline 4500,6500 & $<-2.5$ & -0.6448 & 0.5575 & 0.6678 \\
4500,6500 & $-2.5,-2.0$ & -0.2419 & 0.3023 & 0.2879 \\
4500,6500 & $-2.0,-1.5$ & -0.1947 & 0.3054 & 0.2832 \\
4500,6500 & $-1.5,-1.0$ & -0.1968 & 0.2580 & 0.2673 \\
4500,6500 & $-1.0,-0.5$ & 0.0007 & 0.2260 & 0.1700 \\
4500,6500 & $>-0.5$ & 0.3192 & 0.2566 & 0.3300 \\
\hline
\end{tabular}

included in the linelists will improve the representation of cool stars. An extension to hotter stars will make the model more widely applicable (at present such stars can be filtered out via the PCA reconsuction error). Looking further ahead, the SR approach will form the basis for atmospheric parameter estimation from the very low resolution spectrophotometry $(R \simeq 12-40)$ to be obtained with Gaia (albeit using a more sophisticated and knowledge-based approach to regression, which also includes the accurate parallaxes and high-precision photometry from Gaia). Our pattern recognition approach is probably indispensable in such an application, because the low resolution and spectral purity of the spectrophotometry prevent the definition of traditional indices.

Acknowledgements. This work was partly funded by a DFG Emmy-Noether Nachwuchsgruppe grant to C.A.L. Bailer-Jones. Y.S. Lee, T.C. Beers, and T. Sivarani acknowledge partial support for this work from grant AST 04-06784, as well as from grant PHY 02-16783: Physics Frontiers Center/Joint Institute for Nuclear Astrophysics (JINA), both awarded by the U.S. National Science Foundation.

We wish to thank the referee, Norbert Christlieb, for a careful reading of this manuscript and for his useful remarks.

Funding for the SDSS and SDSS-II has been provided by the Alfred P. Sloan Foundation, the Participating Institutions, the National Science Foundation, the U.S. Department of Energy, the National Aeronautics and Space Administration, the Japanese Monbukagakusho, the Max Planck Society, and the Higher Education Funding Council for England. The SDSS Web Site is http: //www. sdss.org/.

The SDSS is managed by the Astrophysical Research Consortium for the Participating Institutions. The Participating Institutions are the American Museum of Natural History, Astrophysical Institute Potsdam, University of Basel, University of Cambridge, Case Western Reserve University, University of Chicago, Drexel University, Fermilab, the Institute for Advanced Study, the Japan Participation Group, Johns Hopkins University, the Joint Institute for Nuclear Astrophysics, the Kavli Institute for Particle Astrophysics and Cosmology, the Korean Scientist Group, the Chinese Academy of Sciences (LAMOST), Los Alamos National Laboratory, the Max-Planck-Institute for Astronomy (MPIA), the Max-Planck-Institute for Astrophysics (MPA), New Mexico State University, Ohio State University, University of Pittsburgh, University of Portsmouth, Princeton University, the United States Naval Observatory, and the University of Washington.

\section{References}

Abazajian, K., Adelman-McCarthy, J. K., Agüeros, M. A., et al. 2005, AJ, 129, 1755

Adelman-McCarthy, J. K., Agüeros, M. A., Allam, S. S., et al. 2007, ApJS, submitted

Allende Prieto, C., Beers, T. C., Wilhelm, R., et al. 2006, ApJ, 636, 804

Alvarez, R., \& Plez, B. 1998, A\&A, 330, 1109

Asplund, M., Grevesse, N., \& Sauval, A. J. 2005, EAS Publ. Ser., 17, 21

Bailer-Jones, C. A. L. 1996, Ph.D. Thesis

Bailer-Jones, C. A. L. 2000, A\&A, 357, 197

Bailer-Jones, C. A. L., Irwin, M., Gilmore, G., \& von Hippel, T. 1997, MNRAS, 292, 157

Bailer-Jones, C. A. L., Irwin, M., \& von Hippel, T. 1998, MNRAS, 298, 361

Barklem, P. S., \& O’Mara, B. J. 1998, MNRAS, 300, 863

Beers, T. C., Rossi, S. Norris, J. E., Ryan, S. G., \& Shefler, T. 1999, AJ, 117, 981 Beers, T. C., Allende Prieto, C., Wilhelm, R., Yanny, B., \& Newberg. H. J. 2004, PASA, 21, 207 
Beers, T. C., Lee, Y. S., Sivarani, T., et al. 2006, MemSAIt, 77, 1171 Bilir, S., Karaali, S., \& Tuncel, S. 2005, Astron. Nachr., 326, 321 Binney, J., \& Merrifield, M. 1998, Galactic Astronomy (Princeton: Princeton Univ. Press)

Castelli, F., Gratton, R. G., \& Kurucz, R. L. 1997, A\&A, 318, 841

Castelli, F., \& Kurucz, R. L. 2003, IAU Symp., 210, A20

Carraro, G., \& Chiosi, C. 1994, A\&A, 288, 751

Einbeck, J., Evers, L., \& Bailer-Jones, C. A. L. 2007, in Lecture Notes in Computational Science and Engineering, ed. A. Gorban, B. Kegl, D. Wunsch, \& A. Zinovyev (Springer Verlag), in press

Friel, E. D., \& Janes, K. A. 1993, A\&A, 267, 75

Fukugita, M., Ichikawa, T., Gunn, J. E., et al. 1996, AJ, 111, 1748

Girardi, L., Grebel, E. K., Odenkirken, M., \& Chiosi, C. 2004, A\&A, 422, 205

Gulati, R. K., Gupta, R., \& Rao, N. K. 1996, A\&A, 322, 933

Gunn, J. E., Carr, M., Rockosi, C., et al. 1998, AJ, 116, 3040

Gunn, J. E., Siegmund, W. A., Mannery, E. J., et al. 2006, AJ, 131, 2332

Harris, W. E. 1996, AJ, 112, 1487

Hastie, T., Tibshirani, R., \& Friedman, J. 2001 (Springer), The Elements of Statistical Learning

Hogg, D. W., Finkbeiner, D. P., Schlegel, D. J., \& Gunn, J. E. 2001, AJ, 122, 2129

Ivézic, Z., Lupton, R. H., Schlegel, D., et al. 2004, AN, 325, 583

Kupka, F., Piskunov, N., Ryabchikova, T. A., Stemples, H. C., \& Weiss, W. W. 1999, A\&AS, 138, 119

Lázaro, C., Arellano Ferro, A., Arevalo, M. J., et al. 2006, MNRAS, 372, 69
Lee, Y. S., Beers, T. C., Sivarani, T., et al. 2006, BAAS, 38, 168.15 Lee, Y. S., Beers, T. C., Sivarani, T., et al. 2007, AJ, submitted

Lupton, R. H., Gunn, J. E., \& Griffin, R. F. 1987, AJ, 93, 5

McClure, R. D., Forrester, W. T., \& Gibson, J. 1974, ApJ, 189, 409

Newberg, H. J. 2003, BAAS, 35, 1385

Odewahn, S. C., Cohen, S. H., Windhorst, R. A., \& Philip, N. S. 2002, ApJ, 568, 539

Pier, J. R., Munn, J. A., Hindsley, R. B., et al. 2003, AJ, 125, 1559

Plez, B., \& Cohen, J. G. 2005, A\&A, 434, 1117

Salaris, M., Degl'Innocenti, S., \& Weiss, A. 1997, ApJ, 479, 665

Sandage, A. 1970, ApJ, 162, 841

Shetrone, M. D. 1994, PASP, 106, 161

Singh, H. P., Bailer-Jones, C. A. L., \& Gupta, R. 2001, in Automated Data Analysis in Astronomy (New Delhi, India: Narosa Publishing House), 69

Smith, J. A., Tucker, D. L., Kent, S., et al. 2002, AJ, 123, 2121

Smith, V. V., \& Suntzeff, N. B. 1987, AJ, 92, 2

Snider, S., Allende Prieto, C., von Hippel, T., et al. 2001, ApJ, 562, 528

Storrie-Lombardi, M. C., Irwin, M. J., von Hippel, T., \& Storrie-Lombardi, L. J. 1995, Vistas in Astronomy 38, 331

Stoughton, C., Lupton, R. H., Bernardi, M., et al. 2002, AJ, 123, 485

Tianxing, L., \& Janes, K. A. 1987, PASP, 99, 1076

Tucker, D. L., Kent, S., Richmond, M. W., et al. 2006, AN, 327, 821

Willemsen, P. G., Hilker, M., Kayser, A., \& Bailer-Jones, C. A. L. 2005, A\&A, 436, 379

York, D. G., Adelman, J., Anderson, J. E. Jr., et al. 2000, AJ, 120, 1579 